\begin{tabular}{|c|l|}
\hline Title & A dvanced validation of CFD-FDTD combined method using highly applicable solver for reentry blackout prediction \\
\hline Author(s) & Takahashi, Y usuke \\
\hline Citation & $\begin{array}{l}\text { Journal of Physics D: A pplied Physics, 49(1), 015201 } \\
\text { https://doi.org/10.1088/0022-3727/49/1/015201 }\end{array}$ \\
\hline Issue Date & 2015-11-18 \\
\hline Doc URL & http://hdl.handle.net/2115/63601 \\
\hline Type & article(author version) \\
\hline File Information & paper_ramc.pdf \\
\hline
\end{tabular}

Instructions for use 


\title{
Advanced Validation of CFD-FDTD Combined Method using Highly Applicable Solver for Reentry Blackout Prediction
}

\author{
Yusuke Takahashi ${ }^{1}$ \\ Hokkaido University, Kita 13 Nishi 8, Kita-ku, Sapporo, Hokkaido, 060-8628, Japan
}

\begin{abstract}
An analysis model of plasma flow and electromagnetic waves around a reentry vehicle for radio frequency blackout prediction during aerodynamic heating was developed in this study. The model was validated based on experimental results from the radio attenuation measurement program. The plasma flow properties, such as electron number density, in the shock layer and wake region were obtained using a newly developed unstructured grid solver that incorporated real gas effect models and could treat thermochemically non-equilibrium flow. To predict the electromagnetic waves in plasma, a frequency-dependent finite-difference time-domain method was used. Moreover, the complicated behaviour of electromagnetic waves in the plasma layer during atmospheric reentry was clarified at several altitudes. The prediction performance of the combined model was evaluated with profiles and peak values of the electron number density in the plasma layer. In addition, to validate the models, the signal losses measured during communication with the reentry vehicle were directly compared with the predicted results. Based on the study, it was suggested that the present analysis model accurately predicts the radio frequency blackout and plasma attenuation of electromagnetic waves in plasma in communication.
\end{abstract}

\section{Nomenclature}

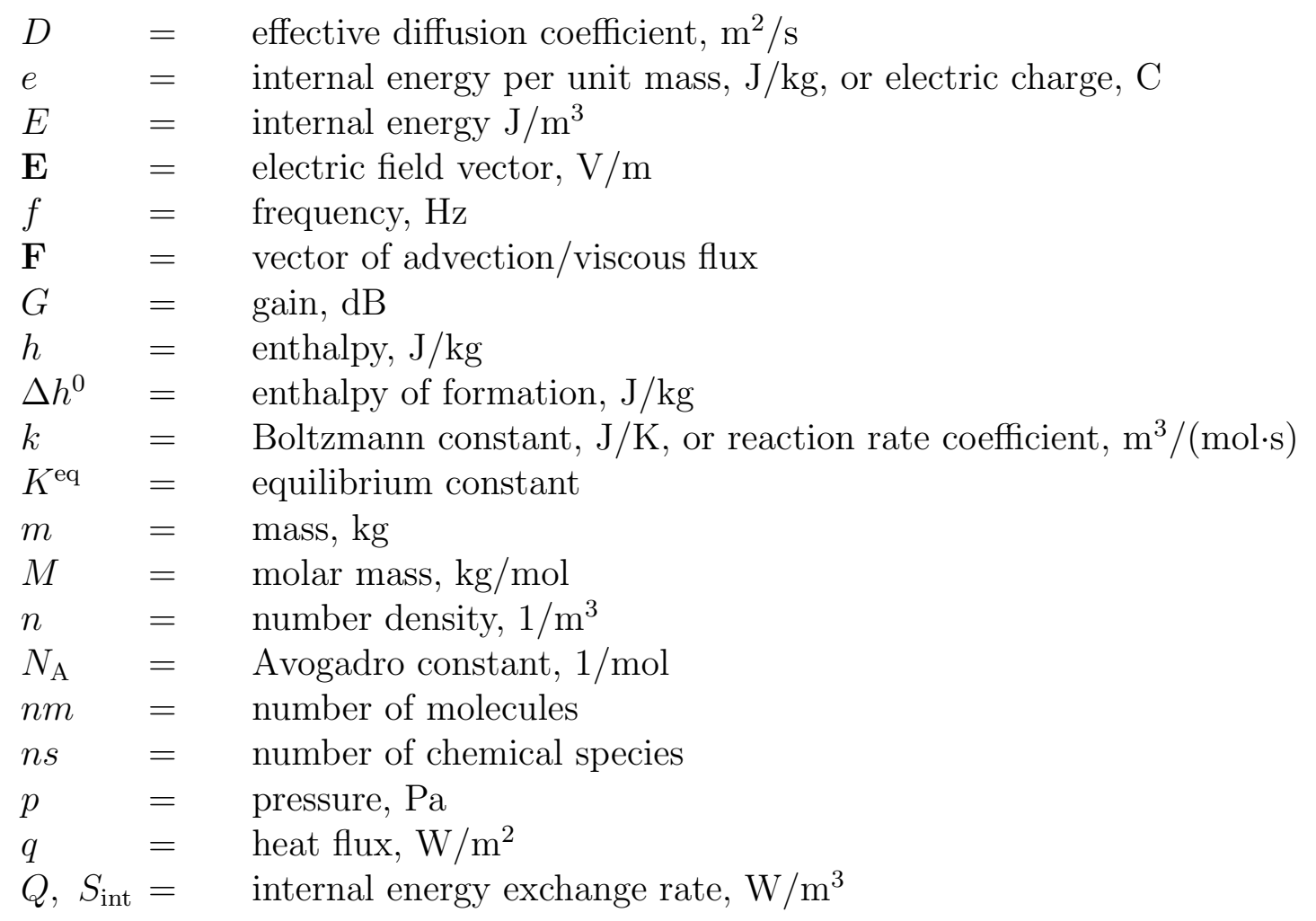

\footnotetext{
${ }^{1}$ Assistant Professor, Faculty of Engineering; ytakahashi@eng.hokudai.ac.jp.
} 


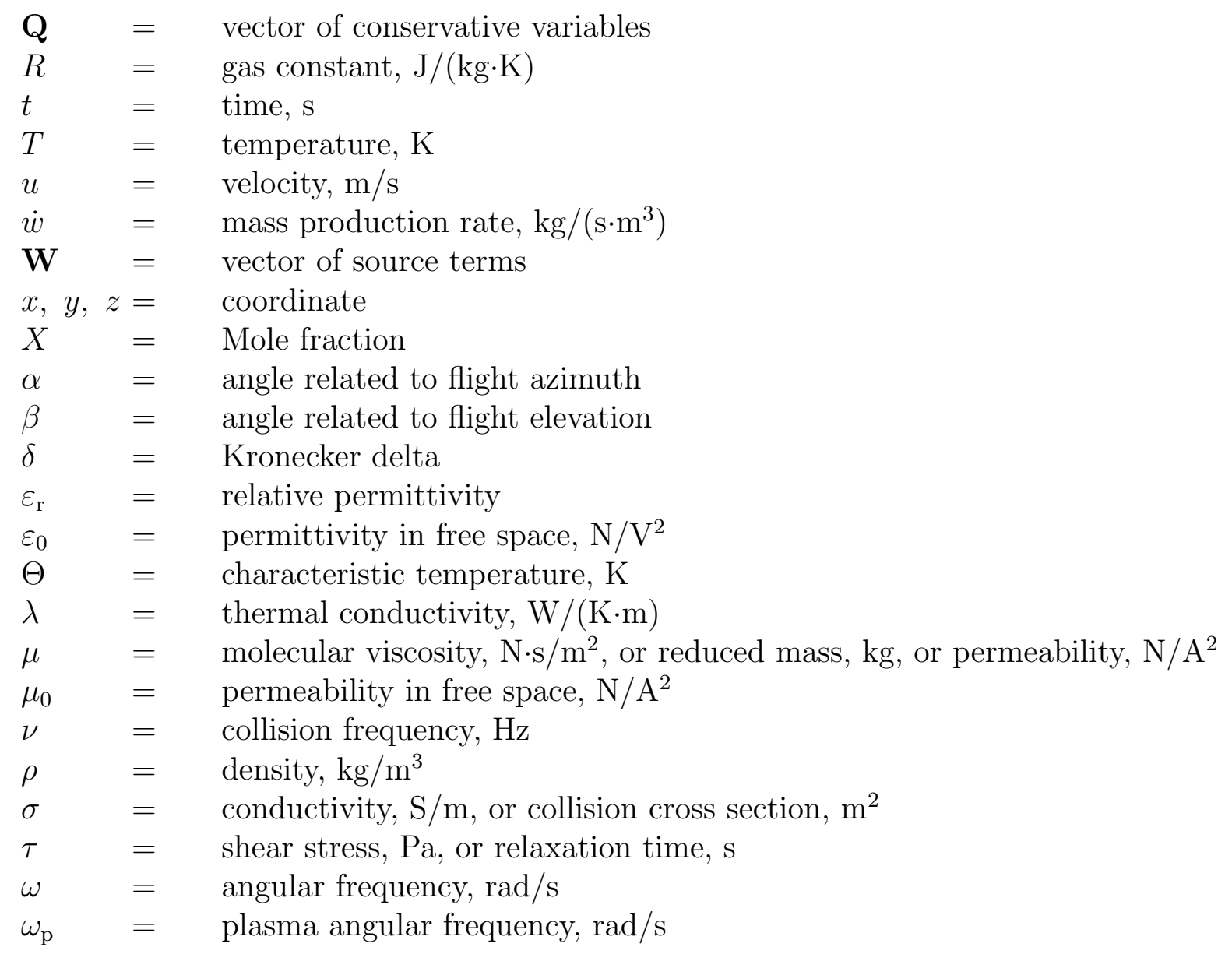

Subscripts

$\begin{array}{lll}\mathrm{b} & = & \text { backward } \\ \mathrm{bd} & = & \text { boundary } \\ \mathrm{c} & = & \text { collision } \\ \mathrm{D} & = & \text { dissociation } \\ \mathrm{ele}, \mathrm{e} & = & \text { electron } \\ \mathrm{f} & = & \text { forward } \\ \mathrm{free} & = & \text { free space } \\ \mathrm{I} & = & \text { ionisation } \\ \mathrm{M} & = & \text { molecular } \\ \mathrm{p} & = & \text { plasma } \\ \mathrm{r} & = & \text { relative } \\ \mathrm{rot}, \mathrm{R} & = & \text { rotation } \\ s & = & \text { species } \\ \mathrm{trs}, \mathrm{T} & = & \text { translation } \\ \mathrm{SL} & = & \text { signal loss } \\ v & = & \text { viscous } \\ \mathrm{vib}, \mathrm{V} & = & \text { vibration }\end{array}$

\section{Superscripts}

$\begin{array}{lll}\mathrm{a} & = & \text { ambipolar } \\ \mathrm{eq} & = & \text { equilibrium } \\ \mathrm{n} & = & \text { time step }\end{array}$




\section{Introduction}

There is a strong demand for the development of technology for recovering samples after landing/splashing down and tracking the position of the vehicle during a reentry mission. An important element of trajectory determination and accurate landing prediction is to keep communications open between the reentry vehicle and the ground station. Currently, lowcost methods, such as the global positioning system (GPS) and Iridium satellite network, are used for communication, but in these methods, which utilize electromagnetic waves, radio frequency $(\mathrm{RF})$ blackouts and plasma attenuation can occur. Because the vehicle's velocity exceeds $7 \mathrm{~km} / \mathrm{s}$ during reentry from a low Earth orbit, dissociated and ionised gases are generated by shock waves at the front of the vehicle and cover the vehicle's surface. When this plasma layer on the surface attenuates or reflects the electromagnetic waves, a radiofrequency (RF) blackout occurs [1].

Several techniques, involving the use of electrophilic substances, magnetic fields, and aerodynamic shaping, have been proposed by various researchers for mitigating the RF blackout problem. For example, signal recoveries of very high frequency (VHF) telemetry and a Cband beacon with water injection as an electrophilic substance were observed in the Gemini 3 mission [2]. Kim et al. [3] reported that plasma density can be controlled and RF blackouts can be reduced by applying a magnetic field on an antenna. In addition, Kundrapu et al. [4] and Usui [5] numerically investigated the possibility of communication during an RF blackout via whistler mode. The vehicle configuration is also an important design parameter to be considered for reducing RF blackouts and plasma attenuation. Belov et al. [6] investigated an aerodynamic shaping technique to alleviate the RF blackouts by mounting slender nosecone probes, containing a communication antenna, ahead of a blunt nose vehicle. Takahashi et al. $[7,8]$ suggested that an inflatable vehicle with a low ballistic coefficient and a large projected area for deploying its flare aeroshell in orbit can reduce the RF blackout. Thus, it may be possible for a vehicle with such features and devices to communicate without RF blackouts during a reentry mission.

A detailed analysis of the reflection and attenuation of electromagnetic waves around the vehicle during the vehicle design process can provide useful information for estimating the possibility of RF blackouts and plasma attenuation. To clarify the behaviour of electromagnetic waves in the plasma flow around the reentry vehicle, computational fluid dynamics (CFD) and computational electromagnetics are effective techniques. Regarding the prediction of electromagnetic waves in a frequency-dependent medium such as plasma, the frequencydependent finite-difference time-domain (FD2TD) method [9] is useful for determining the propagation of microwaves around a rocket plume [10] or that of electromagnetic waves in isotropic cold plasma [11].

For the design and development of reentry vehicles, numerical prediction methods have become important tools for investigating the plasma flow and electromagnetic waves. Takahashi et al. [12] validated a model that combined CFD and FD2TD for the atmospheric reentry demonstrator (ARD) of the European Space Agency (ESA). However, because the angle of attack and the configuration of the ARD were not perfectly reproduced owing to the limitations in the flow field solver and grid generation, the combined model was not suitably validated. With the recent progress, effective flow field solvers and grid generation software applicable to unstructured meshes have become available. For example, "FaSTAR" (Fast 
aerodynamic routine) [13] has been developed by the Japan Aerospace Exploration Agency (JAXA) as a compressible flow solver for three-dimensional unstructured meshes. Moreover, a version of FaSTAR incorporating real gas effects, "RG-FaSTAR", has been developed for aerodynamic heating simulation of reentry vehicles with orbital speeds. Thus, it has become possible to realistically simulate the plasma flow around a vehicle considering various analytical aspects such as angle of attack and complicated configurations. An example of such an analysis is the Radio Attenuation Measurement C (RAM C) project of the National Aeronautics and Space Administration (NASA). Vehicles developed under the RAM C program have acquired various information about the plasma layer and the signal loss profiles of electromagnetic waves used for communication, with a ground station during reentry. The trajectory data of a RAM $\mathrm{C}$ vehicle were reported in detail. These data are suitable for validating the aforementioned simulation tools, which can then be used for mitigating RF blackouts and for effectively designing reentry vehicles by clarifying the behaviour of electromagnetic waves in plasma. In addition, plasma properties obtained in the RAM C project were widely used for developing high-enthalpy flow solvers, and the obtained information is effective in validating analysis models. In the present study, the plasma flow and electromagnetic waves around a RAM $\mathrm{C}$ vehicle are numerically investigated using RG-FaSTAR and a FD2TD simulation code. The predicted results from the numerical simulation are then validated against the experimental data of the RAM $\mathrm{C}$ vehicle.

\section{RAM C Reentry Vehicle}

A brief overview of the RAM project [14] is presented in this section. The objective of the RAM project was to obtain reentry flight data for use in flow field models and propagation models of electromagnetic waves during atmospheric reentry. Three reentry flights were conducted on October 19, 1967 (RAM C-1), August 22, 1968 (RAM C-2), and October 1, 1970 (RAM C-3) by Greenwich Mean Time, with multistage solid-fuel Scout vehicles used to launch the RAM C vehicles from NASA's Wallops Flight Facility, Wallops Island, Virginia.

The RAM C-1, RAM C-2, and RAM C-3 vehicles had blunt cone configurations of $9^{\circ}$ half angle with nose radii of approximately $0.1595,0.1525$, and $0.1595 \mathrm{~m}$, respectively. The schematic configuration of a RAM C vehicle is shown in Fig. 1. A phenolic graphite charring ablator was used for the RAM C-1 and RAM C-3 nose caps as a heat-shield material, whereas a Beryllium heat-sink nose cap was used for RAM C-2. The Beryllium nose cap of the RAM C-2 was ejected at an altitude of approximately $56 \mathrm{~km}$, exposing a Teflon nose. A Teflon ablator was coated on the bodies of all the vehicles for thermal protection. The RAM C-1 and RAM C-3 vehicles injected water, an electrophilic substance, into the plasma layer generated by the shock wave to reduce the electron density level and mitigate RF blackouts. Onboard diagnostic instruments and communication antennas, such as VHF telemetry (225.7 and 259.7 $\mathrm{MHz}$ for RAM C-1, 230.4 and 259.7 MHz for RAM C-3), X-band telemetry $(9,210 \mathrm{MHz})$, and C-band beacon (5,700 MHz for RAM C-1 and 5,800 MHz for RAM C-2 and RAM C-3), were mounted on the vehicles. In addition to the antennas mentioned above, K-band $(35,000$ $\mathrm{MHz}), \mathrm{X}$-band (10,044 MHz), S-band (3,348 MHz), and L-band (1,116 MHz) reflectometers were also installed at four stations on the RAM C-2 vehicle to obtain information about the peak electron density in the plasma layer (see also Fig. 1). To measure the number density of electrons, the three RAM C vehicles were equipped with an electrostatic (Langmuir) probe and a thermocouple probe at the aft.

During the launch, the Scout vehicle with the RAM C-3 payload lifted off vertically, 


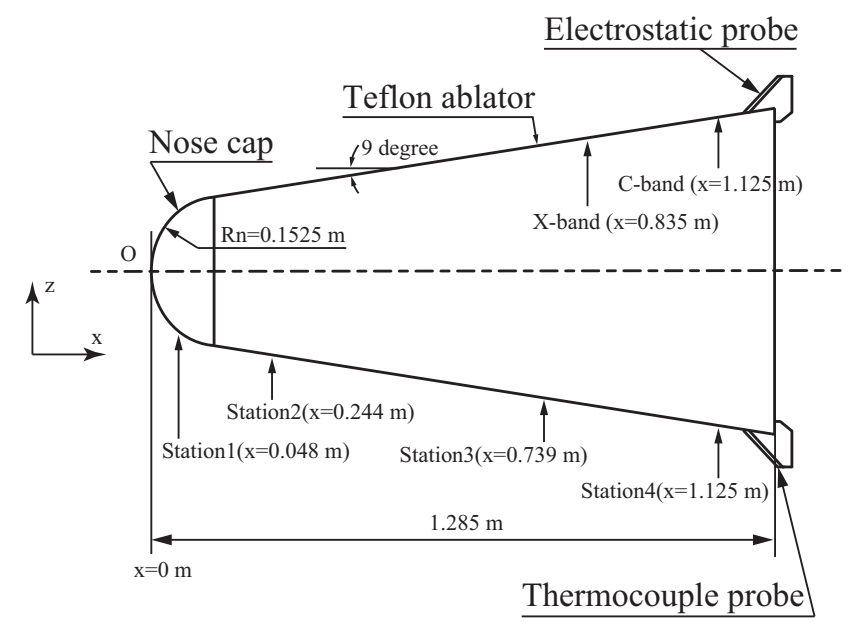

Figure 1: Schematic configuration of RAM C vehicle

and its flight azimuth from the Wallops Flight Facility was recorded as $109^{\circ}$. The RAM C vehicles reached their apogees at an altitude of over $220 \mathrm{~km}$ and then reentered the Earth's atmosphere with a reentry velocity of $7.4 \mathrm{~km} / \mathrm{s}$ after separation from the Scout vehicle. To prevent perturbations due to large angles of attack, the vehicles were rotated about the body axis at approximately 3 revolutions/s. During the free flight phase after separation, a radar at Bermuda tracked the $\mathrm{C}$-band beacon of the RAM $\mathrm{C}$ vehicles. The trajectory parameters of the RAM C-3 reentry, such as altitude, latitude, longitude, velocity, flight azimuth, and flight elevation, have been reported by Weaver and Bowen [15]. The flight path angles of the vehicle during reentry were approximately $-15.0^{\circ}$ for elevation and $116^{\circ}$ for azimuth. During the reentry, the vehicles were deduced to have a maximum angle of attack of $5^{\circ}$ with rotation in the roll direction. Because the geographic coordinates of the Bermuda radar were known $\left(32.348^{\circ}\right.$ latitude and $-64.654^{\circ}$ longitude), the position of the vehicles relative to the Bermuda radar could be easily determined. Figure 2 shows the angles at which the radar was visible from the viewpoint of the vehicle with respect to the flight azimuth and elevation during reentry. Considering the body axis $\left(x^{\prime} y^{\prime} z^{\prime}\right), \alpha$ is the angle between the $x^{\prime}$ axis and the direction of the radar projected on the $x^{\prime}-y^{\prime}$ plane, and $\beta$ is the angle between the $z^{\prime}$ axis and the radar direction.

\section{Flow Field Model}

In this section, the governing equations, thermochemical non-equilibrium models, and numerical implementation for plasma flow simulation used in this study are described.

\subsection{Governing Equations}

The flow field is assumed to be laminar and continuum. To express the thermochemical nonequilibrium flow, the finite-rate chemical reaction and four-temperature models are employed here. In the four-temperature model, the translational $\left(T_{\text {trs }}\right)$, rotational $\left(T_{\text {rot }}\right)$, vibrational $\left(T_{\text {vib }}\right)$, and electron $\left(T_{\text {ele }}\right)$ temperatures are considered, and the electron-excitation temperature is assumed to be equilibrated with the electron temperature. The flow field is described by the Navier-Stokes equations with a multi-temperature model that includes the species 


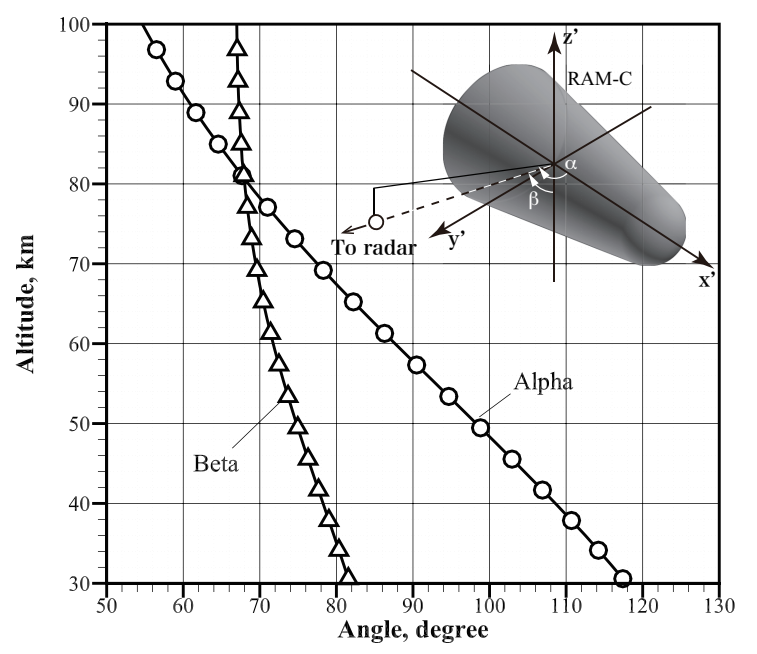

Figure 2: Angle profile of Bermuda radar position with respect to flight azimuth and elevation during atmospheric reentry

mass and internal energies (rotation, vibration, and electron energies) conservations in addition to the total mass, momentum, and total energy conservations. The Navier-Stokes equation system is expressed as follows:

$$
\frac{\partial \mathbf{Q}}{\partial t}+\frac{\partial \mathbf{F}}{\partial x_{j}}=\frac{\partial \mathbf{F}_{\mathrm{v}}}{\partial x_{j}}+\mathbf{W}
$$

where the conservative vector is expressed as $\mathbf{Q}=\left[\rho, \rho u_{i}, E, \rho_{s}, E_{\text {rot }}, E_{\text {vib }}, E_{\text {ele }}\right]^{t}$. The advection and viscous flux vectors and the source-term vector in Eq. (1) can be expressed as

$$
\mathbf{F}=\left[\begin{array}{c}
\rho u_{j} \\
\rho u_{i} u_{j}+\delta_{i j} p \\
(E+p) u_{j} \\
\rho_{s} u_{j} \\
E_{\mathrm{rot}} u_{j} \\
E_{\mathrm{vib}} u_{j} \\
E_{\mathrm{ele}} u_{j}
\end{array}\right], \mathbf{F}_{\mathrm{v}}=\left[\begin{array}{c}
0 \\
\tau_{i j} \\
q_{j}+\rho \sum_{s=1}^{n s} h_{s} D_{s} \frac{\partial X_{s}}{\partial x_{j}}+u_{i} \tau_{i j} \\
\rho D_{s} \frac{\partial X_{s}}{\partial x_{j}} \\
q_{\mathrm{rot}, j}+\rho \sum_{s=M}^{n m} e_{\mathrm{rot}, s} D_{s} \frac{\partial X_{s}}{\partial x_{j}} \\
q_{\mathrm{vib}, j}+\rho \sum_{s=M}^{n m} e_{\mathrm{vib}, s} D_{s} \frac{\partial X_{s}}{\partial x_{j}} \\
q_{\mathrm{ele}, j}+\rho h_{\mathrm{ele}} D_{\text {ele }} \frac{\partial X_{\text {ele }}}{\partial x_{j}}-p_{\text {ele }} u_{j}
\end{array}\right], \mathbf{W}=\left[\begin{array}{c}
0 \\
0 \\
0 \\
\dot{w}_{s} \\
S_{\text {int,rot }} \\
S_{\text {int,vib }} \\
S_{\text {int,ele }}
\end{array}\right],
$$

where $\delta_{i, j}, S_{\text {int }}$, and $\dot{w}_{s}$ denote the Kronecker delta, internal energy exchange rate, and mass production rate, respectively. Note that the subscript " $s$ " in the equations represents each chemical species, and " $M$ " indicates the set of molecules considered $\left(M=\mathrm{N}_{2}, \mathrm{O}_{2}, \mathrm{NO}, \mathrm{N}_{2}^{+}\right.$, $\mathrm{O}_{2}^{+}$, and $\mathrm{NO}^{+}$). The stress tensor $\tau_{i j}$ and heat flux $q_{j}$ in Eq. (2) can be expressed as follows:

$$
\tau_{i j}=\mu\left(\frac{\partial u_{i}}{\partial x_{j}}+\frac{\partial u_{j}}{\partial x_{i}}\right)-\frac{2}{3} \mu \frac{\partial u_{k}}{\partial x_{k}} \delta_{i j}
$$




$$
q_{j}=q_{\mathrm{trs}, j}+q_{\mathrm{rot}, j}+q_{\mathrm{vib}, j}+q_{\mathrm{ele}, j}=\lambda_{\mathrm{trs}} \frac{\partial T_{\mathrm{trs}}}{\partial x_{j}}+\lambda_{\mathrm{rot}} \frac{\partial T_{\mathrm{rot}}}{\partial x_{j}}+\lambda_{\mathrm{vib}} \frac{\partial T_{\mathrm{vib}}}{\partial x_{j}}+\lambda_{\text {ele }} \frac{\partial T_{\mathrm{ele}}}{\partial x_{j}} .
$$

The equation of state is given by

$$
\begin{aligned}
& p=\sum_{s \neq \mathrm{e}}^{n s} \rho_{s} R_{s} T_{\mathrm{trs}}+p_{\text {ele }} \\
& p_{\text {ele }}=\rho_{\mathrm{e}} R_{\mathrm{e}} T_{\text {ele }}
\end{aligned}
$$

The total internal energy can be defined as

$$
E=E_{\text {trs }}+E_{\text {rot }}+E_{\text {vib }}+E_{\text {ele }}+\sum_{s=1}^{n s} \rho_{s} \Delta h_{s}^{0}+\frac{1}{2} \rho u_{i} u_{i} .
$$

The translational, rotational, vibrational, and electron energies are given by

$$
\begin{aligned}
& E_{\mathrm{trs}}=\sum_{s \neq \mathrm{e}}^{n s} \frac{3}{2} \rho_{s} R_{s} T_{\mathrm{trs}}, \\
& E_{\mathrm{rot}}=\sum_{s=\mathrm{M}}^{n m} \rho_{s} R_{s} T_{\mathrm{rot}}, \\
& E_{\mathrm{vib}}=\sum_{s=\mathrm{M}}^{n m} \frac{\rho_{s} R_{s} \Theta_{\mathrm{vib}, s}}{\exp \left(\Theta_{\mathrm{vib}, s} / T_{\mathrm{vib}}\right)-1}, \\
& E_{\text {ele }}=\frac{3}{2} \rho_{\mathrm{e}} R_{\mathrm{e}} T_{\text {ele }},
\end{aligned}
$$

where $\Delta h_{s}^{0}$ and $\Theta_{\mathrm{vib}, s}$ represent the enthalpy of formation and vibrational characteristic temperatures, respectively.

\subsection{Transport Properties}

To evaluate the viscosity, thermal conductivity, and binary diffusion coefficients in the viscous terms of the Navier-Stokes equations, Yos's formula [16] based on the first Chapman-Enskog approximation is applied. The effective diffusion coefficients, $D_{s}$, are calculated using binary diffusion coefficients obtained by Curtiss and Hirschfelder's formula [17]. To determine the first-order transport properties, the collision cross-sections between two chemical species are required. In this study, the collision cross-sections are obtained from Gupta's work [18], except for the e-N and e-O pairs, which are calculated using the Fertig model [19,20]. The ambipolar diffusion is expressed as follows:

$$
D_{s}^{a}=\left(1+\frac{T_{\text {ele }}}{T_{\mathrm{trs}}}\right) D_{s}
$$

\subsection{Chemical Reaction Models}

As shown in Table 1 , the reaction system is considered to comprise 11 chemical species $\left(\mathrm{N}_{2}\right.$, $\mathrm{O}_{2}, \mathrm{NO}, \mathrm{N}_{2}^{+}, \mathrm{O}_{2}^{+}, \mathrm{NO}^{+}, \mathrm{N}, \mathrm{O}, \mathrm{N}^{+}, \mathrm{O}^{+}$, and $\mathrm{e}^{-}$) and 49 chemical reactions in the hightemperature region. The forward reaction rate is calculated using the following expression:

$$
k_{\mathrm{f}}\left(T_{\mathrm{f}}\right)=C_{r} T_{\mathrm{f}}^{n_{r}} \exp \left(-\frac{\theta_{r}}{T_{\mathrm{f}}}\right) .
$$


The reaction rate coefficients $C_{r}, n_{r}$, and $\theta_{r}$ are obtained from Park's report [21], whereas the backward reaction rate is obtained using the forward reaction rate and the equilibrium constant, as follows:

$$
k_{\mathrm{b}}\left(T_{\mathrm{b}}\right)=\frac{k_{\mathrm{f}}\left(T_{\mathrm{b}}\right)}{K^{\mathrm{eq}}\left(T_{\mathrm{b}}\right)} .
$$

The effective forward and backward temperatures $T_{\mathrm{f}}$ and $T_{\mathrm{b}}$ are determined according to $T_{\text {trs }}$ and $T_{\text {ele }}$ or the geometric average of $T_{\text {trs }}, T_{\text {rot }}, T_{\text {vib }}$, and $T_{\text {ele }}$. The equilibrium constants are calculated using the curve-fit formula reported by Park [22]. For a charge-exchange reaction between the molecular nitrogen and the ionised nitrogen $(r=49$ in Table 1$)$, the curve-fit parameters are determined according to Gupta's work [18].

\subsection{Internal Energy Exchange Models}

The energy transfer between each pair of internal energy modes is considered, including the translation-rotation (T-R), translation-vibration (T-V), translation-electron (T-e), rotationvibration $(\mathrm{R}-\mathrm{V})$, rotation-electron (R-e), and vibration-electron (V-e). Thus, the internal energy exchange rates $S_{\text {int }}$ in the source term vector $\mathbf{W}$ can be expressed as follows:

$$
\begin{aligned}
& S_{\text {int,rot }}=Q_{\mathrm{T}-\mathrm{R}}-Q_{\mathrm{R}-\mathrm{V}}-Q_{\mathrm{R}-\mathrm{e}}+Q_{\mathrm{D}}^{\text {rot }}, \\
& S_{\text {int,vib }}=Q_{\mathrm{T}-\mathrm{V}}+Q_{\mathrm{R}-\mathrm{V}}+Q_{\mathrm{e}-\mathrm{V}}+Q_{\mathrm{D}}^{\text {vib }}, \\
& S_{\text {int,ele }}=Q_{\mathrm{T}-\mathrm{e}}+Q_{\mathrm{R}-\mathrm{e}}-Q_{\mathrm{e}-\mathrm{V}}+Q_{\mathrm{D}}^{\text {ele }}+Q_{\mathrm{I}}^{\text {ele }} .
\end{aligned}
$$

\subsubsection{Translational-Rotational Energy Exchange}

The translational-rotational energy exchange rate $Q_{\mathrm{T}-\mathrm{R}}$ is given by Parker's work [23] as

$$
Q_{\mathrm{T}-\mathrm{R}}=\sum_{s=M}^{n m} \frac{E_{\mathrm{rot}}^{\mathrm{eq}}\left(T_{\mathrm{trs}}\right)-E_{\mathrm{rot}}\left(T_{\mathrm{rot}}\right)}{\left\langle\tau_{s}\right\rangle_{\mathrm{T}-\mathrm{R}}} .
$$

In Eq. (18), $\left\langle\tau_{s}\right\rangle$ is given by

$$
\left\langle\tau_{s}\right\rangle_{\mathrm{T}-\mathrm{R}}=\frac{Z_{\mathrm{rot}, s} \sum_{k \neq \mathrm{e}}^{n s} \tau_{s, k}}{\left[1+\frac{\pi^{\frac{3}{2}}}{2}\left(\frac{\Theta_{\mathrm{rot}, s}}{T_{\mathrm{trs}}}\right)^{\frac{1}{2}}+\left(\frac{\pi^{2}}{4}+\pi\right)\left(\frac{\Theta_{\mathrm{rot}, s}}{T_{\mathrm{trs}}}\right)\right]},
$$

where the relaxation time in a collision between chemical species $s$ and $k$ is expressed as

$$
\tau_{s, k}=\left[n_{k} \sigma_{\mathrm{rot}}\left(\frac{8 k N_{\mathrm{A}} T_{\mathrm{trs}}}{\pi \mu_{s, k}}\right)^{\frac{1}{2}}\right]^{-1} .
$$

In Eq. (20), $\mu_{s, k}$ represents the reduced mass of species $s$ and $k$, given by

$$
\mu_{s, k}=M_{s} M_{k} /\left(M_{s}+M_{k}\right) .
$$

In the present study, the effective collision cross-section is set as $\sigma_{\text {rot }}=10^{-19} \mathrm{~m}^{2}$. In addition, $\Theta_{\text {rot }, s}$ and $Z_{\text {rot }, s}$ denote the rotational characteristic temperatures and constants for each chemical species, which are summarised in Table 2. It is assumed that the constants of ionic species are the same as those of the neutral one. 
Table 1: Chemical reactions

\begin{tabular}{|c|c|c|c|c|c|c|c|c|}
\hline$r$ & Forward & & Backward & $\overline{C_{r}}$ & $n_{r}$ & $\theta_{r}$ & $\overline{T_{\mathrm{f}}}$ & $T_{\mathrm{b}}$ \\
\hline $1-6$ & $\mathrm{~N}_{2}+M_{1}$ & $\rightleftharpoons$ & $\mathrm{N}+\mathrm{N}+M_{1}$ & $7.0 \times 10^{21}$ & -1.60 & 113,200 & $T_{\mathrm{f} 1}$ & $T_{\text {trs }}$ \\
\hline $7-10$ & $\mathrm{~N}_{2}+M_{2}$ & $\rightleftharpoons$ & $\mathrm{N}+\mathrm{N}+M_{2}$ & $3.0 \times 10^{22}$ & -1.60 & 113,200 & $T_{\mathrm{f} 1}$ & $T_{\mathrm{trs}}$ \\
\hline 11 & $\mathrm{~N}_{2}+\mathrm{e}^{-}$ & $\rightleftharpoons$ & $\mathrm{N}+\mathrm{N}+\mathrm{e}^{-}$ & $1.2 \times 10^{25}$ & -1.60 & 113,200 & $T_{\text {ele }}$ & $T_{\mathrm{b} 1}$ \\
\hline $12-17$ & $\mathrm{O}_{2}+M_{1}$ & $\rightleftharpoons$ & $\mathrm{O}+\mathrm{O}+M_{1}$ & $2.0 \times 10^{21}$ & -1.50 & 59,500 & $T_{\mathrm{f} 1}$ & $T_{\mathrm{trs}}$ \\
\hline $18-21$ & $\mathrm{O}_{2}+M_{2}$ & $\rightleftharpoons$ & $\mathrm{O}+\mathrm{O}+M_{2}$ & $1.0 \times 10^{22}$ & -1.50 & 59,500 & $T_{\mathrm{f} 1}$ & $T_{\mathrm{trs}}$ \\
\hline $22-27$ & $\mathrm{NO}+M_{1}$ & $\rightleftharpoons$ & $\mathrm{N}+\mathrm{O}+M_{1}$ & $5.0 \times 10^{15}$ & 0.00 & 00 & $T_{\mathrm{f} 1}$ & $T_{\mathrm{trs}}$ \\
\hline $28-31$ & $\mathrm{O}+\mathrm{M}_{2}$ & $\rightleftharpoons$ & $\mathrm{N}+\mathrm{O}+M_{2}$ & $1.1 \times 10^{17}$ & 0.00 & 75,500 & $T_{\mathrm{f} 1}$ & $T_{\text {trs }}$ \\
\hline 32 & $\mathrm{NO}+\mathrm{O}$ & $\rightleftharpoons$ & $\mathrm{N}+\mathrm{O}_{2}$ & $8.4 \times 10^{12}$ & 0.00 & 19,450 & $T_{\mathrm{trs}}$ & $T_{\mathrm{trs}}$ \\
\hline 33 & $\mathrm{~N}_{2}+\mathrm{O}$ & $\rightleftharpoons$ & $\mathrm{NO}+\mathrm{N}$ & $6.4 \times 10^{17}$ & -1.00 & 38 & $T_{\mathrm{trs}}$ & $T_{\mathrm{trs}}$ \\
\hline 34 & $\mathrm{~N}+\mathrm{N}$ & $\rightleftharpoons$ & $\mathrm{N}_{2}^{+}+\mathrm{e}^{-}$ & $4.4 \times 10^{7}$ & 1.50 & 67,500 & $T_{\mathrm{trs}}$ & $T_{\mathrm{trs}}$ \\
\hline 35 & $+\mathrm{O}$ & $\rightleftharpoons$ & $\mathrm{O}_{2}^{+}+\mathrm{e}^{-}$ & $7.1 \times 10^{2}$ & 2.7 & 80 & $T_{\mathrm{trs}}$ & $T_{\mathrm{trs}}$ \\
\hline 36 & $+\mathrm{O}$ & $\rightleftharpoons$ & $\mathrm{NO}^{+}+\mathrm{e}^{-}$ & $8.8 \times 10^{8}$ & 1.00 & 31,900 & $T_{\mathrm{trs}}$ & $T_{\mathrm{trs}}$ \\
\hline 37 & $\mathrm{~N}+\mathrm{e}^{-}$ & $\rightleftharpoons$ & $\mathrm{N}^{+}+\mathrm{e}^{-}+\mathrm{e}^{-}$ & $2.5 \times 10^{34}$ & -3 & 00 & $T_{\text {ele }}$ & $T_{\text {ele }}$ \\
\hline 38 & $\mathrm{O}+\mathrm{e}^{-}$ & $\rightleftharpoons$ & $\mathrm{O}^{+}+\mathrm{e}^{-}+\mathrm{e}^{-}$ & $3.9 \times 10^{33}$ & -3.78 & 158,500 & $T_{\text {ele }}$ & $T_{\text {ele }}$ \\
\hline 39 & $\mathrm{NO}^{+}+\mathrm{O}$ & $\rightleftharpoons$ & $\mathrm{N}^{+}+\mathrm{O}_{2}$ & $1.0 \times 10^{12}$ & 0.50 & 77,200 & $T_{\mathrm{trs}}$ & $T_{\mathrm{trs}}$ \\
\hline 40 & $\mathrm{O}_{2}^{+}+\mathrm{N}$ & $\rightleftharpoons$ & $\mathrm{N}^{+}+\mathrm{O}_{2}$ & $8.7 \times 10^{13}$ & 0.14 & 28,600 & $T_{\mathrm{trs}}$ & $T_{\mathrm{trs}}$ \\
\hline 41 & $\mathrm{O}^{+}+\mathrm{NO}$ & $\rightleftharpoons$ & $\mathrm{N}^{+}+\mathrm{O}_{2}$ & $1.4 \times 10^{5}$ & 1.90 & 26,600 & $T_{\mathrm{trs}}$ & $T_{\mathrm{trs}}$ \\
\hline 42 & $\mathrm{O}_{2}^{+}+\mathrm{N}_{2}$ & $\rightleftharpoons$ & $\mathrm{N}_{2}^{+}+\mathrm{O}_{2}$ & $9.9 \times 10^{12}$ & 0.00 & 40,700 & $T_{\mathrm{trs}}$ & $T_{\mathrm{trs}}$ \\
\hline 43 & $\mathrm{O}_{2}^{+}+\mathrm{O}$ & $\rightleftharpoons$ & $\mathrm{O}^{+}+\mathrm{O}_{2}$ & $4.0 \times 10^{12}$ & -0.09 & 18,000 & $T_{\mathrm{trs}}$ & $T_{\mathrm{trs}}$ \\
\hline 44 & $\mathrm{NO}^{+}+\mathrm{N}$ & $\rightleftharpoons$ & $\mathrm{O}^{+}+\mathrm{N}_{2}$ & $3.4 \times 10^{13}$ & -1.08 & 12 & $T_{\mathrm{trs}}$ & $T_{\mathrm{trs}}$ \\
\hline 45 & $\mathrm{NO}^{+}+\mathrm{O}_{2}$ & $\rightleftharpoons$ & $\mathrm{O}_{2}^{+}+\mathrm{NO}$ & $2.4 \times 10^{13}$ & 0.41 & 32,600 & $T_{\mathrm{trs}}$ & $T_{\mathrm{trs}}$ \\
\hline 46 & $\mathrm{NO}^{+}+\mathrm{O}$ & $\rightleftharpoons$ & $\mathrm{O}_{2}^{+}+\mathrm{N}$ & $7.2 \times 10^{12}$ & 0.29 & 48,600 & $T_{\mathrm{trs}}$ & $T_{\mathrm{trs}}$ \\
\hline 47 & $\mathrm{O}^{+}+\mathrm{N}_{2}$ & $\rightleftharpoons$ & $\mathrm{N}_{2}^{+}+\mathrm{O}$ & $9.1 \times 10^{11}$ & 0.36 & 22,800 & $T_{\mathrm{trs}}$ & $T_{\mathrm{trs}}$ \\
\hline 48 & $\mathrm{NO}^{+}+\mathrm{N}$ & $\rightleftharpoons$ & $\mathrm{N}_{2}^{+}+\mathrm{O}$ & $7.2 \times 10^{13}$ & 0.00 & 35,500 & $T_{\mathrm{trs}}$ & $T_{\text {trs }}$ \\
\hline 49 & $\mathrm{~N}_{2}+\mathrm{N}^{+}$ & $\rightleftharpoons$ & $\mathrm{N}_{2}^{+}+\mathrm{N}$ & $1.0 \times 10^{12}$ & 0.50 & 12,200 & $T_{\mathrm{trs}}$ & $T_{\mathrm{trs}}$ \\
\hline
\end{tabular}

$$
\begin{aligned}
& M_{1}=\mathrm{N}_{2}, \mathrm{O}_{2}, \mathrm{NO}, \mathrm{N}_{2}^{+}, \mathrm{O}_{2}^{+}, \mathrm{NO}^{+} \\
& M_{2}=\mathrm{N}, \mathrm{O}, \mathrm{N}^{+}, \mathrm{O}^{+} \\
& T_{\mathrm{f} 1}=\sqrt[3]{T_{\mathrm{trs}} T_{\mathrm{rot}} T_{\mathrm{vib}}} \\
& T_{\mathrm{b} 1}=\sqrt{T_{\mathrm{trs}} T_{\mathrm{ele}}}
\end{aligned}
$$


Table 2: $\Theta_{\text {rot }, s}$ and $Z_{\mathrm{rot}, s}$ for each species

\begin{tabular}{ccccccc}
\hline \hline Species & $\mathrm{N}_{2}$ & $\mathrm{O}_{2}$ & $\mathrm{NO}$ & $\mathrm{N}_{2}^{+}$ & $\mathrm{O}_{2}^{+}$ & $\mathrm{NO}^{+}$ \\
\hline$\Theta_{\text {rot }, \mathrm{s}}$ & 2.879 & 2.069 & 2.440 & 2.879 & 2.069 & 2.440 \\
$\mathrm{Z}_{\mathrm{rot}, \mathrm{s}}$ & 15.70 & 14.40 & 15.05 & 15.70 & 14.40 & 15.05 \\
\hline \hline
\end{tabular}

\subsubsection{Translational, Rotational-Vibrational Energy Exchange}

The translational-vibrational relaxation can be described by the Landau-Teller equation. According to Park [24], the energy exchange between the vibrational and rotational modes also needs to be considered. The formula of Millikan and White [25], along with Park's collision-limiting correction [26] at high temperatures, is used for the translational-vibrational coupling. On the other hand, the rotational-vibrational relaxation time is evaluated using only the Millikan-White formula. Thus, the vibrational relaxation rate $Q_{\mathrm{TR}-\mathrm{V}}$ is represented as follows:

$$
Q_{\mathrm{TR}-\mathrm{V}}=\sum_{s=M}^{n m}\left[0.6 \frac{E_{\mathrm{vib}}^{\mathrm{eq}}\left(T_{\mathrm{trs}}\right)-E_{\mathrm{vib}}\left(T_{\mathrm{vib}}\right)}{\left\langle\tau_{s}^{\mathrm{MW}}\right\rangle+\left\langle\tau_{s}^{\mathrm{P}}\right\rangle}+0.4 \frac{E_{\mathrm{vib}}^{\mathrm{eq}}\left(T_{\mathrm{rot}}\right)-E_{\mathrm{vib}}\left(T_{\mathrm{vib}}\right)}{\left\langle\tau_{s}^{\mathrm{MW}}\right\rangle}\right] .
$$

In the above equation, the first and second terms represent the rotational-vibrational and translational-vibrational energy transfer rates, respectively. In order to conserve the total energy, the translational energy exchange rate $Q_{\mathrm{T}-\mathrm{V}}$ and rotational energy exchange rate $Q_{\mathrm{R}-\mathrm{V}}$ are expressed as

$$
\begin{aligned}
Q_{\mathrm{T}-\mathrm{V}} & =\sum_{s=M}^{n m}\left[0.6 \frac{E_{\mathrm{vib}}^{\mathrm{eq}}\left(T_{\mathrm{trs}}\right)-E_{\mathrm{vib}}\left(T_{\mathrm{vib}}\right)}{\left\langle\tau_{s}^{\mathrm{MW}}\right\rangle+\left\langle\tau_{s}^{\mathrm{P}}\right\rangle}\right], \\
Q_{\mathrm{R}-\mathrm{V}} & =\sum_{s=M}^{n m}\left[0.4 \frac{E_{\mathrm{vib}}^{\mathrm{eq}}\left(T_{\mathrm{rot}}\right)-E_{\mathrm{vib}}\left(T_{\mathrm{vib}}\right)}{\left\langle\tau_{s}^{\mathrm{MW}}\right\rangle}\right] .
\end{aligned}
$$

For the temperature range $300-8,000 \mathrm{~K}$, the relaxation time is given by a semi-empirical equation introduced by Millikan and White as follows:

$$
\tau_{s, k}^{\mathrm{MW}}=(p / 101,325)^{-1} \exp \left(A_{s, k} T_{\mathrm{trs}}^{-1 / 3}-B_{s, k}\right),
$$

where

$$
\begin{aligned}
A_{s, k} & =1.16 \times 10^{-3} \mu_{s, k}{ }^{1 / 2} \Theta_{\mathrm{vib}, s}^{4 / 3} \\
B_{s, k} & =0.015 \mu_{s, k}^{1 / 4} A_{s, k}+18.42 \\
\mu_{s, k} & =M_{s} M_{k} /\left(M_{s}+M_{k}\right) .
\end{aligned}
$$

Note that the unit of $M$ in Eq. (25) is $\mathrm{g} / \mathrm{mol}$. The mean relaxation time of the species is given by

$$
\left\langle\tau_{s}^{\mathrm{MW}}\right\rangle=\frac{\sum_{k=M}^{n m} \frac{\rho_{k}}{M_{k}}}{\sum_{k \neq \mathrm{e}}^{n s} \frac{\rho_{k}}{M_{k} \tau_{s, k}^{\mathrm{MW}}} .}
$$


Moreover, Park's collision-limiting correction of the relaxation time is evaluated as

$$
\left\langle\tau_{s}^{\mathrm{P}}\right\rangle=\left[n_{s} \sigma_{\mathrm{vib}}\left(\frac{8 R_{s} T_{\mathrm{trs}}}{\pi}\right)^{1 / 2}\right]^{-1},
$$

where $\sigma_{\mathrm{vib}}$ represents the effective collision cross-section, which is set as $\sigma_{\mathrm{vib}}=1.0 \times 10^{-21}\left(50,000 / T_{\mathrm{trs}}\right)^{2}$ $\mathrm{m}^{2}$.

\subsubsection{Translational-Electron Energy Exchange}

Appleton's energy-exchange model [27] is used for the coupling between the translational and electron energy modes:

$$
Q_{\mathrm{T}-\mathrm{e}}=\sum_{s \neq \mathrm{e}}^{n s} 2 \nu_{\mathrm{e}, s} \frac{3}{2} \rho_{\mathrm{e}} R_{s}\left(T_{\mathrm{trs}}-T_{\mathrm{ele}}\right),
$$

where $\nu_{\mathrm{e}, s}$ is the collision frequency between the electron and the other species $s$. According to Mitchner and Kruger [28], the effective collision frequency between the electron and the ionic species is given in SI units as

$$
\begin{aligned}
\nu_{\mathrm{e}, s} & =3.64 \times 10^{-6} n_{\mathrm{s}} \ln (\Lambda) / T_{\mathrm{ele}}^{3 / 2}, \\
\Lambda & =1.24 \times 10^{7}\left(T_{\mathrm{e}}^{3} / n_{\mathrm{ele}}\right)^{1 / 2} .
\end{aligned}
$$

According to Gnoffo's work [29], for collisions between an electron and a neutral species, the effective collision frequency is given by

$$
\nu_{\mathrm{e}, s}=n_{s} \sigma_{\mathrm{e}, s}\left(\frac{8 R_{\mathrm{e}} T_{\mathrm{ele}}}{\pi}\right)^{1 / 2} .
$$

The effective cross-section $\sigma_{\mathrm{e}, s}$ is obtained from a curve fit as

$$
\sigma_{\mathrm{e}, s}=\tilde{a}_{s}+\tilde{b}_{s} T_{\text {ele }}+\tilde{c}_{s} T_{\text {ele }}^{2}
$$

where the coefficients $\tilde{a}_{s}, \tilde{b}_{s}$, and $\tilde{c}_{s}$ are listed in Table 3 .

Table 3: Curve-fit constants for $\sigma_{\mathrm{e}, s}$

\begin{tabular}{cccccc}
\hline \hline Species & $\mathrm{N}_{2}$ & $\mathrm{O}_{2}$ & $\mathrm{NO}$ & $\mathrm{N}$ & $\mathrm{O}$ \\
\hline$\tilde{a}_{s}$ & $7.5 \times 10^{-20}$ & $2.0 \times 10^{-20}$ & $1.0 \times 10^{-19}$ & $5.0 \times 10^{-20}$ & $1.2 \times 10^{-20}$ \\
$\tilde{b}_{s}$ & $5.5 \times 10^{-24}$ & $6.0 \times 10^{-24}$ & 0 & 0 & $1.7 \times 10^{-24}$ \\
$\tilde{c}_{s}$ & $-1.0 \times 10^{-28}$ & 0 & 0 & 0 & $-2.0 \times 10^{-29}$ \\
\hline \hline
\end{tabular}

\subsubsection{Rotational-Electron Energy Exchange}

In Nishida and Matsumoto's work [30], the rate of the rotational-electron energy coupling is given as follows:

$$
Q_{\mathrm{R}-\mathrm{e}}=\sum_{s=\mathrm{M}}^{n m} 2 \nu_{\mathrm{e}, s} g_{\mathrm{rot}, s} \frac{3}{2} \rho_{\mathrm{e}} R_{s}\left(T_{\text {rot }}-T_{\text {ele }}\right) .
$$

In the present calculation, the coefficients $g_{\mathrm{rot}, s}=10$ are assumed for the neutral species such as $\mathrm{N}_{2}, \mathrm{O}_{2}$, and $\mathrm{NO}$ [31]. For the ionic molecules, because accurate values are unavailable, the same coefficients are used as those for the neutral species. 


\subsubsection{Vibrational-Electron Energy Exchange}

The energy transfer between the vibrational and electron energy modes is given by

$$
Q_{\mathrm{e}-\mathrm{V}}=\sum_{s=\mathrm{M}}^{n m} \frac{E_{\mathrm{vib}}^{\mathrm{eq}}\left(T_{\mathrm{ele}}\right)-E_{\mathrm{vib}}\left(T_{\mathrm{vib}}\right)}{\left\langle\tau_{s}\right\rangle_{\mathrm{e}-\mathrm{V}}} .
$$

The relaxation time $\left\langle\tau_{s}\right\rangle_{\mathrm{e}-\mathrm{V}}$ is obtained using Lee's work [32]. Because the translationalelectron energy couplings for $\mathrm{O}_{2}$ and $\mathrm{NO}$ are slower than that for $\mathrm{N}_{2}$, the relaxation times for $\mathrm{O}_{2}$ and $\mathrm{NO}$ are obtained by multiplying that for $\mathrm{N}_{2}$ by 300, as proposed by Park and Lee [33].

\subsubsection{Energy Loss due to Chemical Reactions}

The energy losses in the vibrational and rotational modes due to the chemical reactions $Q_{\mathrm{D}}^{\text {rot }}$ and $Q_{\mathrm{D}}^{\mathrm{vib}}$ represent the dissociation energies of the heavy-particle impact reactions. These can be given by a non-preferential dissociation model [29] as

$$
\begin{aligned}
Q_{\mathrm{D}}^{\mathrm{rot}} & =\sum_{s=M}^{n m} e_{\mathrm{rot}} \dot{w}_{s}^{\mathrm{D}}, \\
Q_{\mathrm{D}}^{\mathrm{vib}} & =\sum_{s=M}^{n m} e_{\mathrm{vib}} \dot{w}_{s}^{\mathrm{D}},
\end{aligned}
$$

where $\dot{w}_{s}^{D}$ is the mass production rate of the heavy-particle impact dissociations $(r=1-10$, 12-31 in Table 1). Moreover, $e_{\text {rot }}$ and $e_{\text {vib }}$ represent the rotational and vibrational energies per unit mass, respectively.

On the other hand, the electron energy loss due to the electron-impact dissociation/ionisation reactions $Q_{\mathrm{D}}^{\text {ele }}$ and $Q_{\mathrm{I}}^{\text {ele }}$ are given by

$$
\begin{aligned}
Q_{\mathrm{D}}^{\mathrm{e}} & =E_{\mathrm{D}, \mathrm{N}_{2}} \dot{w}_{\mathrm{N}_{2}}^{\mathrm{e}}, \\
Q_{\mathrm{I}}^{\mathrm{e}} & =E_{\mathrm{I}, \mathrm{N}} \dot{w}_{\mathrm{N}}^{\mathrm{e}}+E_{\mathrm{I}, \mathrm{O}} \dot{w}_{\mathrm{O}}^{\mathrm{e}},
\end{aligned}
$$

where $\dot{w}_{\mathrm{N}_{2}}^{\mathrm{e}}, \dot{w}_{\mathrm{N}}^{\mathrm{e}}$, and $\dot{w}_{\mathrm{O}}^{\mathrm{e}}$ are the mass production rates of the electron-impact dissociation and ionisations $(r=11,37$, and 38 in Table 1$)$, respectively. In addition, $E_{\mathrm{D}, \mathrm{N}_{2}}$ represents the dissociation energy of molecular nitrogen, and $E_{\mathrm{I}, \mathrm{N}}$ and $E_{\mathrm{I}, \mathrm{O}}$ represent the ionisation energies of atomic nitrogen and atomic oxygen, respectively.

\subsection{Simulation Code: RG-FaSTAR}

The system of Navier-Stokes equations is converted into the delta form and solved using a finite-volume approach. The flow properties are defined at the centre of a control volume in the flow-field simulation. The numerical fluxes of the advection term in the equations are calculated using the simple low-dissipation advection upstream splitting method (SLAU) scheme [34] with the monotonic upstream-centred scheme for the interpolation of the conservation laws (MUSCL) for the second order of spatial accuracy. On the other hand, the viscous terms of the equation system are calculated using the second-order central-difference method. The spatial gradients of the flow properties are calculated using the Green-Gauss 


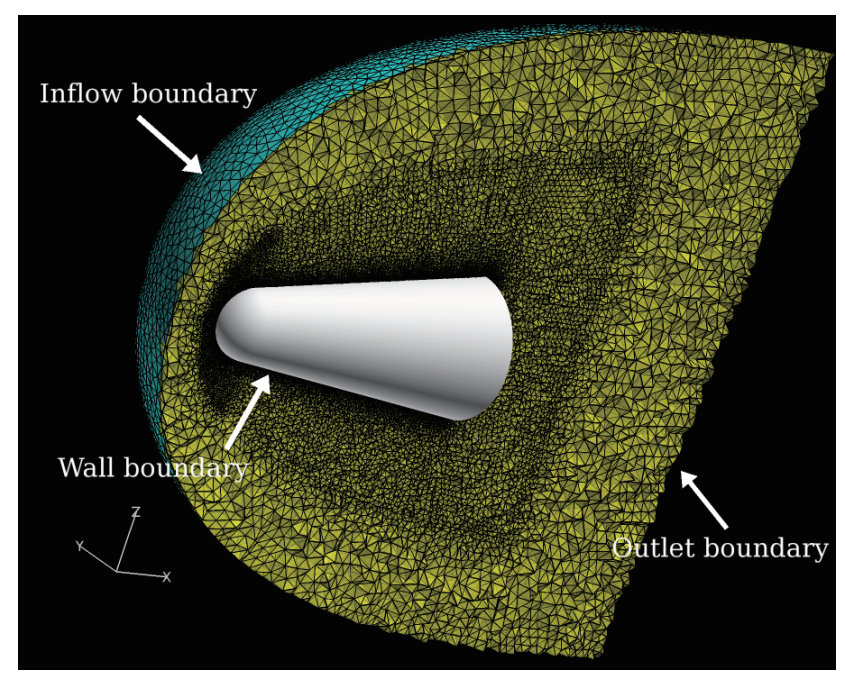

Figure 3: Computational grid system and boundary conditions for RG-FaSTAR

method. A time integration is implicitly performed using a time-marching method. As a matrix solver for robust and effective time integration in the thermochemical non-equilibrium flow simulation, the lower-upper symmetric Gauss-Seidel (LU-SGS) method [35] is combined with the point-implicit method [36].

These physical and numerical models are implemented in the newly developed highenthalpy flow solver RG-FaSTAR. This flow solver is a version of the fast unstructured CFD code FaSTAR [13], originally developed by JAXA, that incorporates real gas effects. These solvers can handle unstructured grid systems, such as tetrahedral, triangular prism, square pyramid, and hexahedral meshes, and have high flexibility in generating the computational grid. For massive parallel computation on a high-performance computer, the message passing interface (MPI) technique with a domain partition approach is adopted.

In RG-FaSTAR, single-temperature, two-temperature, and four-temperature models are available. In the present flow field simulation, the two-temperature model, in which the translational temperature is equilibrated to the rotational one and the electron temperature is equilibrated to the vibrational one, is used.

\subsection{Boundary and Calculation Conditions}

The computational grid system and boundary conditions are shown in Fig. 3. At the inflow boundary, the freestream velocity, density, and temperature are determined according to the orbit data. All the flow properties at the outlet boundary are determined by the $0^{\text {th }}$ extrapolation. The non-slip condition and the non-catalytic condition at the wall boundary are imposed for the flow velocity and mass concentration, respectively. In addition, the pressure gradient normal to the wall is assumed to be 0 . The translational temperatures at the wall boundary are fixed at $273 \mathrm{~K}$ as a cooling wall condition, and the electron temperature is set to be adiabatic.

Four analysis cases with altitudes between 80 and $50 \mathrm{~km}$ are considered here. The input parameters, i.e., the previously mentioned freestream parameters, are set according to the flight conditions, as shown in Table 4 . The freestream velocity is set according to the study by Weaver and Bowen [15].

As shown in Fig. 3, the grid system is composed of tetrahedral and triangular prism cells 
Table 4: Freestream parameters as computational conditions

\begin{tabular}{c|cccc}
\hline \hline Altitude, km & 80 & 70 & 60 & 50 \\
\hline Density, kg/m & $1.80 \times 10^{-5}$ & $8.83 \times 10^{-5}$ & $3.40 \times 10^{-4}$ & $1.15 \times 10^{-3}$ \\
Temperature, K & 195.6 & 210.9 & 242.0 & 265.3 \\
Velocity, m/s & 7400 & 7400 & 7400 & 7400 \\
\hline \hline
\end{tabular}

to capture the steep gradients of the flow properties in the boundary layer. The numbers of elements and nodes in the grid are 13.6 million and 3.3 million, respectively. It was reported by Jones and Cross [37] that the RAM $\mathrm{C}$ vehicle flew with a nonzero angle of attack motion (less than $5^{\circ}$ ) and a roll motion of 3 revolutions/s about the body axis during the atmospheric reentry. In the present simulations, the angle of attack is set as $5^{\circ}$ for all cases.

\section{Electromagnetic Wave Model}

The behaviour of electromagnetic waves are described by Maxwell's equations. Because the FD2TD method with the first Drude dispersion used in the present study was formulated by Takahashi et al. [8,12], physical and analytical models are briefly described in this section.

The relative permittivity $\left(\varepsilon_{\mathrm{r}}\right)$ and dielectric conductivity $(\sigma)$ in terms of the complex relative permittivity $\left(\varepsilon_{\mathrm{r}}^{*}\right)$ are obtained using the angular frequency $(\omega)$ of the electromagnetic waves, plasma angular frequency $\left(\omega_{\mathrm{p}}\right)$, and collision frequency $\left(\nu_{\mathrm{c}}\right)$. These physical properties $\left(\omega_{\mathrm{p}}\right.$ and $\left.\nu_{\mathrm{c}}\right)$ required in the present FD2TD are transposed from the computational results obtained by a RG-FaSTAR code. On the other hand, for simplicity, the magnetic permeability $(\mu)$ in the FD2TD computational domain is set to the free-space value $\left(\mu_{0}\right)$. In the FD2TD method, Maxwell's equations are discretized on the Yee cell, which is basically constructed with rectangular cells. Thus, the computational domain for the electromagnetic waves becomes a cuboid. For the computational domain, the length in the $x$ direction, width in the $y$ direction, and height in the $z$ direction are $2.25,2.0$, and $2.0 \mathrm{~m}$, respectively. The number of computational grid nodes is set as $500 \times 300 \times 300$ in the $x, y$, and $z$ directions, respectively. The RAM $\mathrm{C}$ vehicle is included in the computational domain, and the origin of the RG-FaSTAR computational domain coincides with that of the electromagnetic-wave calculation domain. For the faces of the computational domain, Mur's first-order absorption boundary condition [38] is imposed.

This analysis model is implemented using an electromagnetic wave simulation code developed by Hokkaido University. For fast computation and efficient allocation of adequate memory, the FD2TD simulation code is parallelised using MPI and OpenMP techniques. A data transfer tool between the electromagnetic wave simulation code and RG-FaSTAR is also prepared, and both the simulation codes using the CFD and FD2TD methods are packaged.

\section{Results and Discussion}

\subsection{Plasma Flow Properties}

The electron number density is influenced by the shock wave in an aerodynamic heating environment, and its distribution strongly affects the propagation of the electromagnetic 


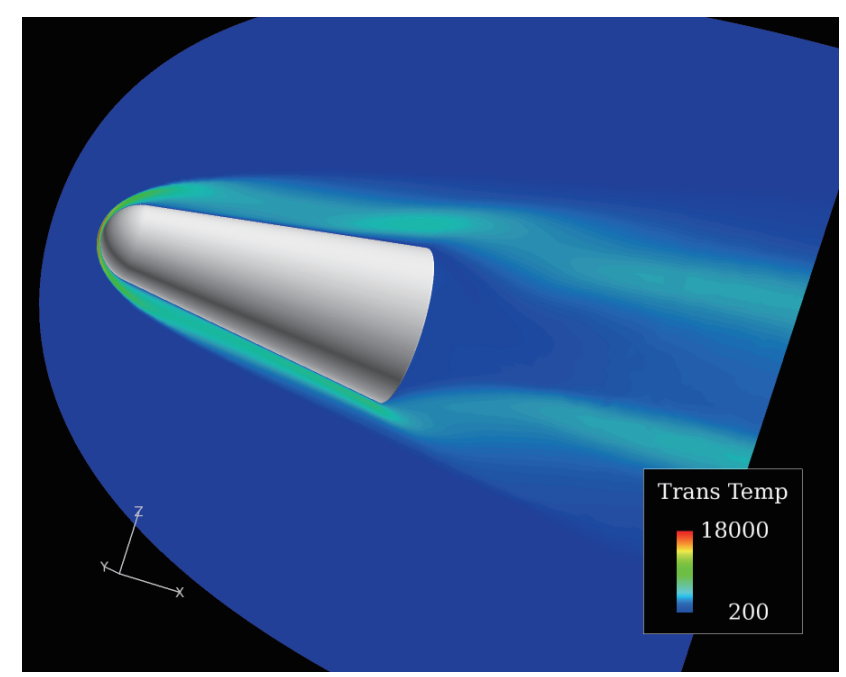

(a) Translational temperature

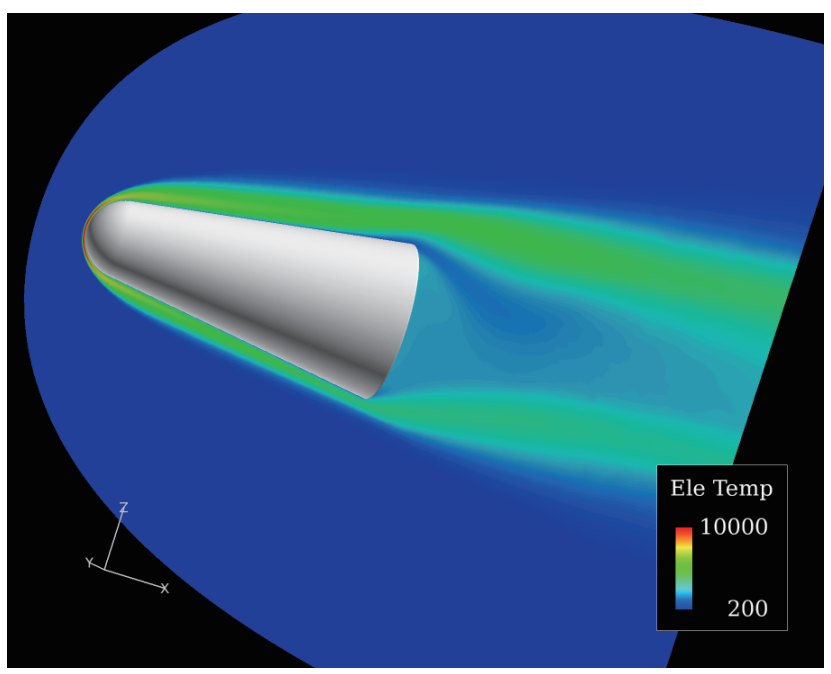

(b) Electron temperature

Figure 4: Distributions of translational and electron temperatures around RAM C vehicle at altitude of $70 \mathrm{~km}$

waves. In this section, we clarify the distributions of the electron number densities using the CFD technique and then validate the present numerical model by comparing its results with the measured data obtained in the RAM C flight experiments.

Figures 4(a) and 4(b) show the distributions of the translational and electron temperatures, respectively, around the RAM $\mathrm{C}$ vehicle at an altitude of $70 \mathrm{~km}$. Because the RAM C vehicle reenters the atmosphere with high velocity, a strong shock wave is generated around the vehicle, and high-temperature plasma is created behind the shock wave. The translational and electron temperatures reach peak values of approximately $18,000 \mathrm{~K}$ and 10,000 K, respectively. It is confirmed that the plasma flow field is in a strong thermal non-equilibrium state because of the low density of the air at this altitude.

The electrons generated near the RAM $\mathrm{C}$ vehicle in the temperature range behind the shock wave are mainly attributed to the associative ionisations $(r=34-36$ in Table 1$)$. On the other hand, the contribution of electron-impact ionisation reactions $(r=37$ and $r=$ 38 in Table 1) to electron generation is relatively small because of the lower temperature in the shock layer. The distribution of the number density of electrons around the RAM $\mathrm{C}$ vehicle at an altitude of $70 \mathrm{~km}$ is illustrated in Fig. 5 in the common logarithmic scale. The electrons mainly distribute in the front region and lateral sides of the RAM C vehicles, whereas the number density of electrons becomes low behind the aft of the vehicle. The cutoff plasma frequency, which is the threshold to reflect electromagnetic waves, can generally be calculated using the electron number density. The link frequencies of the C-band and Xband telemetry horns on the RAM $\mathrm{C}$ vehicle were 5.80 and $9.21 \mathrm{GHz}$, respectively, and thus, the critical number densities for these telemetries are determined as $4.17 \times 10^{17} 1 / \mathrm{m}^{3}$ (C-band) and $1.05 \times 10^{18} 1 / \mathrm{m}^{3}$ (X-band). The number density of electrons is high in the shock layer at its front. In wake region of the RAM $\mathrm{C}$ vehicle rear, the electrons decrease below the critical number density by rarefaction of plasma. Therefore, no electromagnetic waves will be reflected in this region, but attenuation by the conductive medium in which the electromagnetic waves propagate may occur.

In the RAM C flight experiments, the profiles of number density of electrons near the aft of the vehicle were measured by the onboard electrostatic probes. The RAM C-3 vehicle 


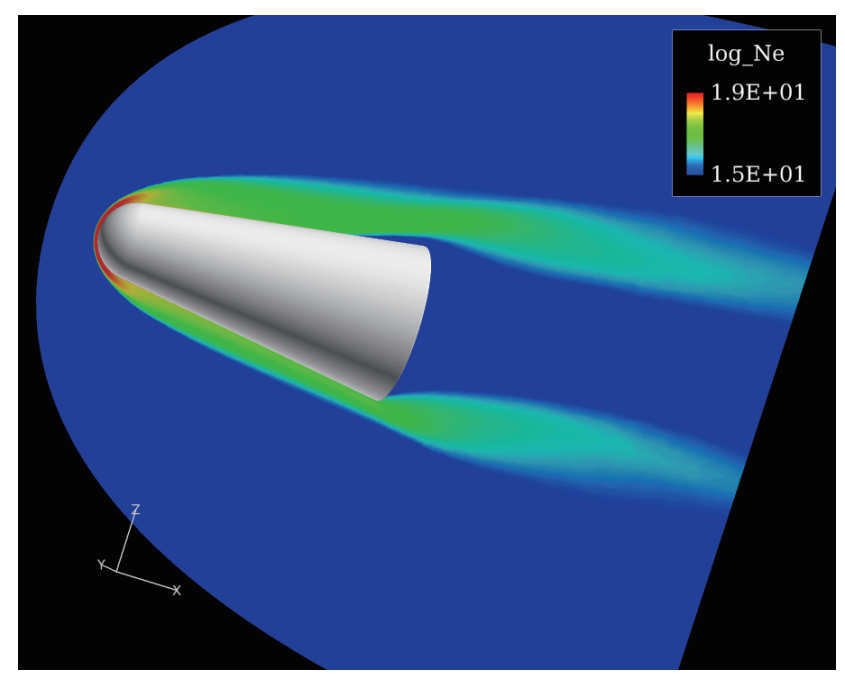

Figure 5: Electron number density distribution around RAM C vehicle at altitude of 70km

injected water from its nose into the flow field around the vehicle body between the altitudes of $85 \mathrm{~km}$ and $35 \mathrm{~km}$. Water injected into the plasma generally decreases the electron density and reduces RF blackouts. However, according to Jones and Cross [37], the effect of water injection at high altitudes, such $80 \mathrm{~km}$ and $70 \mathrm{~km}$, was low. Thus, the electron number density data measured by the probes indicated a similar trend as the results from RAM C-2, which did not inject water into the plasma flow field. In the present study, the electron number density profiles measured by the RAM C-3 vehicle are adopted for comparison with the numerical solutions. Figure 6(a) and 6(b) show the comparisons of the electron number densities measured by the electrostatic probe in the flight experiment and predicted by CFD at altitudes of $80 \mathrm{~km}$ and $70 \mathrm{~km}$, respectively. The data measured by the electrostatic probes are time-averaged values with the error bars representing the electron density fluctuation due to pitching motion. In the present simulations, the electron number densities at the upper and lower surfaces of the vehicle reach minimum and maximum values, respectively, because of the angle of attack of $5^{\circ}$ at each altitude. Therefore, an intermediate value between the electron number densities on the upper and lower surfaces is most likely. From the comparison, the predicted electron number density adequately reproduces the trend of the measured data except in the near-wall region. Because the surface of the RAM C vehicle was coated with the Teflon ablator for thermal protection, it is possible that the gas injected from the Teflon ablation covered the body during atmospheric reentry. In general, the ablation gas can change the thermal environment and chemical compositions in the boundary layer. This possibly suggests that flow field simulation considering ablation gas is also required in order to predict the distribution of number density of electrons accurately.

To diagnose the flow field around the RAM C-2 vehicle during atmospheric reentry, the microwave reflectometer technique was used in the flight experiment. Fifteen antennas, i.e. K-band $(35.000 \mathrm{GHz}), \mathrm{X}$-band $(10.044 \mathrm{GHz})$, S-band $(3.348 \mathrm{GHz})$, and L-band $(1.116 \mathrm{GHz})$ reflectometers, were mounted at four stations on the surface $(x=0.048,0.244,0.739$, and $1.125 \mathrm{~m}$ ). The critical number density of electrons on the reflectometer were determined using the reflectivity of electromagnetic waves by plasma at the reflectometer locations. Figures $7(\mathrm{a}), 7(\mathrm{~b})$, and 7(c) show comparisons of the electron number densities measured by the reflectometer technique in the flight experiment and the peak electron number densities in the normal direction to the vehicle surface predicted by CFD. The black symbols show 


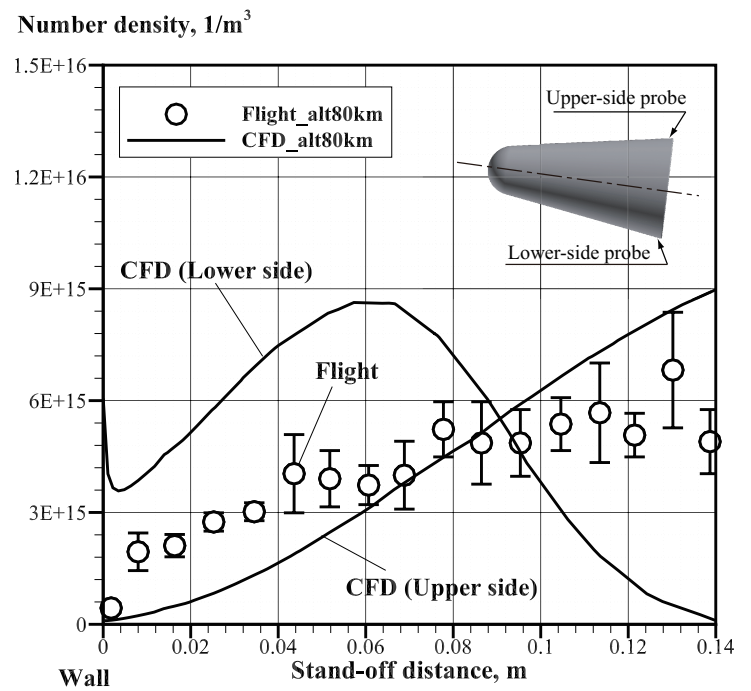

(a) Altitude of $80 \mathrm{~km}$.

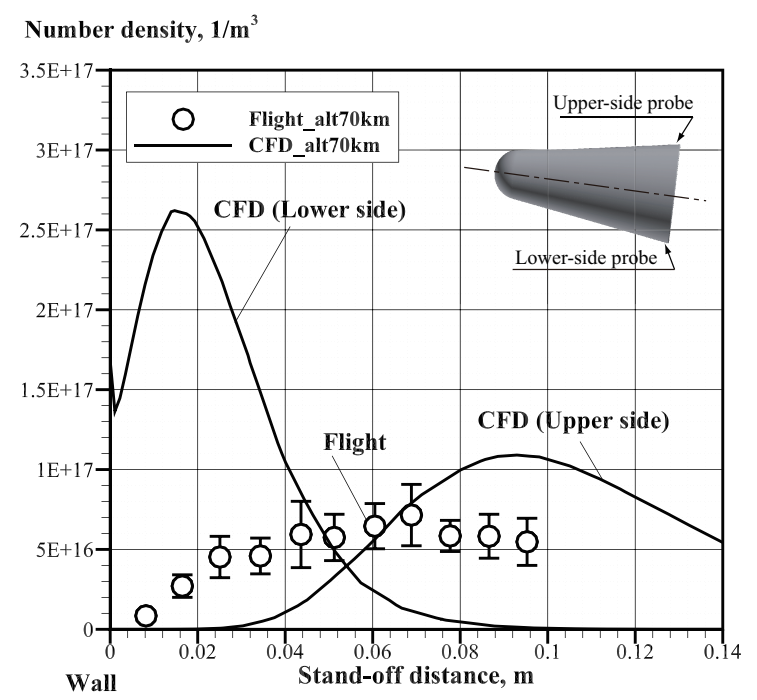

(b) Altitude of $70 \mathrm{~km}$.

Figure 6: Comparison of electron number densities measured by electrostatic probe in flight experiment and predicted by CFD

the experiment data, the white symbols indicate the CFD simulation results, and the grey symbols illustrate the predicted peak electron densities in the shock layer, except for the boundary layer. As mentioned above, because the angle of attack is given for the calculation conditions, the number densities of electrons become peak-to-peak values at the lower and upper surfaces. The likelihood electron density can appear as the intermediate value. At higher altitudes such as $80 \mathrm{~km}$ and $70 \mathrm{~km}$, the electron number densities at the boundary layer are larger than those at the shock layer in the present numerical simulation model, as shown by the grey symbols in Figs. 7(a) and 7(b). However, the peak electron densities in the shock layer are close to the measured data, except in the boundary layer. As discussed in the comparison of electron densities measured by the electrostatic probes, this discrepancy can be attributed to the ablation injected into the plasma flow field from the vehicle surface, which is not treated in the present model. There are several uncertainties that inhibit the development of physical and numerical models related to the ablation. In addition, the numerical cost tends to become large because of the increase in chemical species and chemical reactions considered. Therefore, ablation gas simulation is left for one of the future studies.

\subsection{Electromagnetic Wave Propagation}

In this section, the behaviour of the electromagnetic waves in the plasma media is investigated using the FD2TD method. Then, the computed signal losses for the communication between the RAM $\mathrm{C}$ vehicle and the ground station are directly compared with experimental data.

In general, electromagnetic waves are perfectly reflected on the surface of highly dense plasma whose permittivity $(\varepsilon)$ is negative. Thus, the distribution of the permittivity around the reentry vehicle provides useful information regarding the behaviour of the electromagnetic waves in the plasma. In the present study, the permittivity is calculated using the plasma angular frequency $\left(\omega_{\mathrm{p}}\right)$ and collision frequency $\left(\nu_{\mathrm{c}}\right)$, as follows:

$$
\varepsilon(\omega)=\varepsilon_{0}\left(1-\frac{\omega_{\mathrm{p}}^{2}}{\omega^{2}+\nu_{\mathrm{c}}^{2}}\right) .
$$




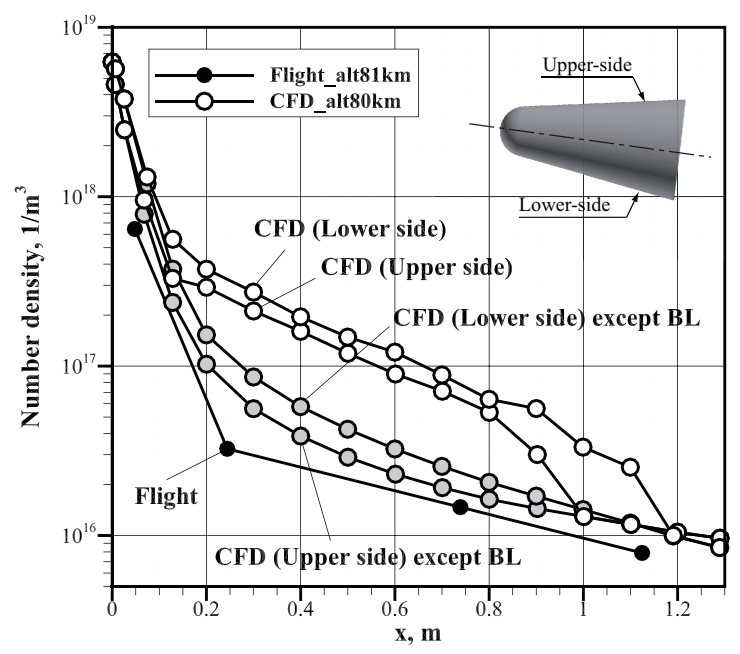

(a) Altitude of $80 \mathrm{~km}$.

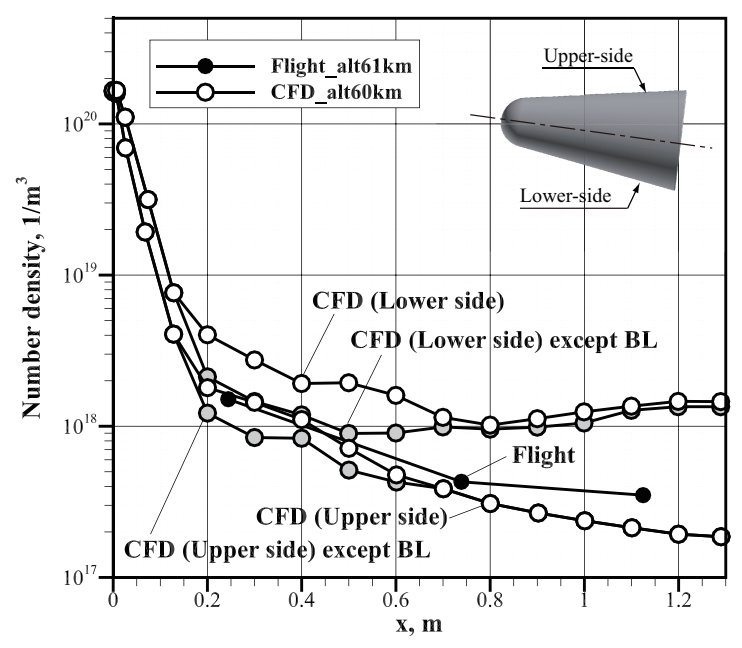

(c) Altitude of $60 \mathrm{~km}$.

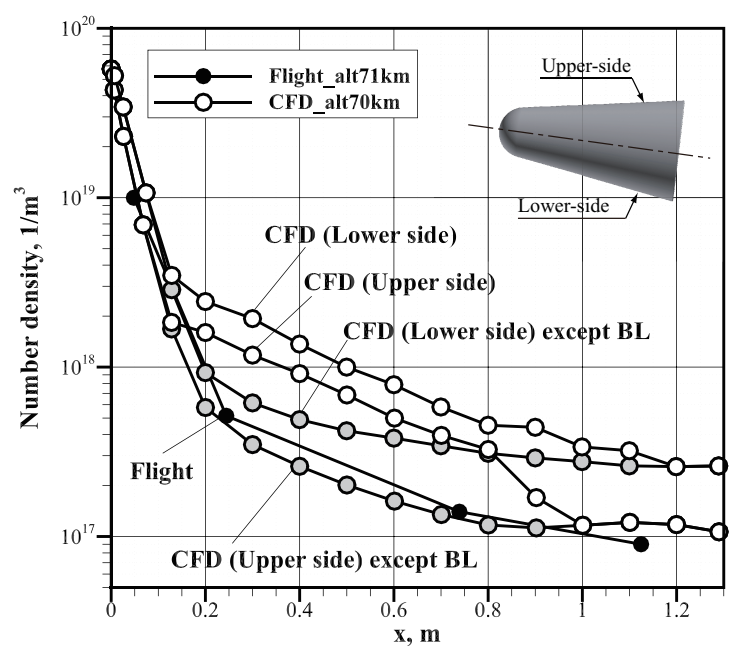

(b) Altitude of $70 \mathrm{~km}$.

Figure 7: Comparison of electron number densities measured by reflectometer technique in flight experiment and peak electron number densities in normal direction to the vehicle surface predicted by CFD 


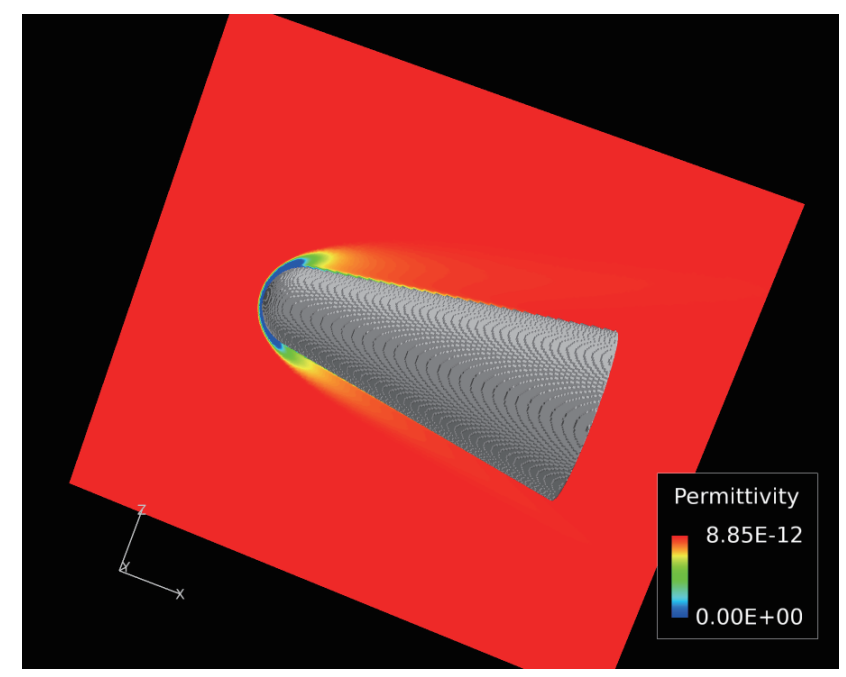

(a) altitude $80 \mathrm{~km}$.

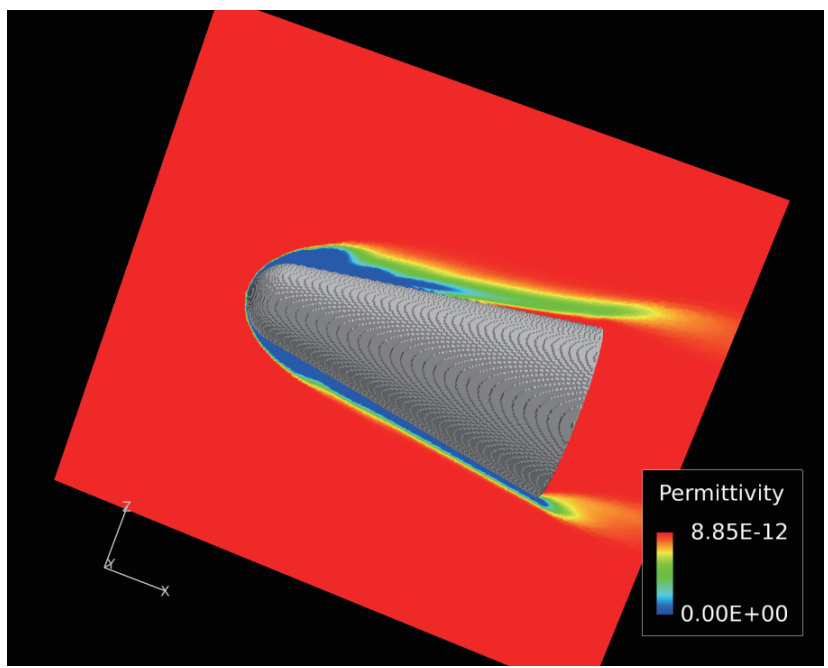

(c) altitude $60 \mathrm{~km}$.

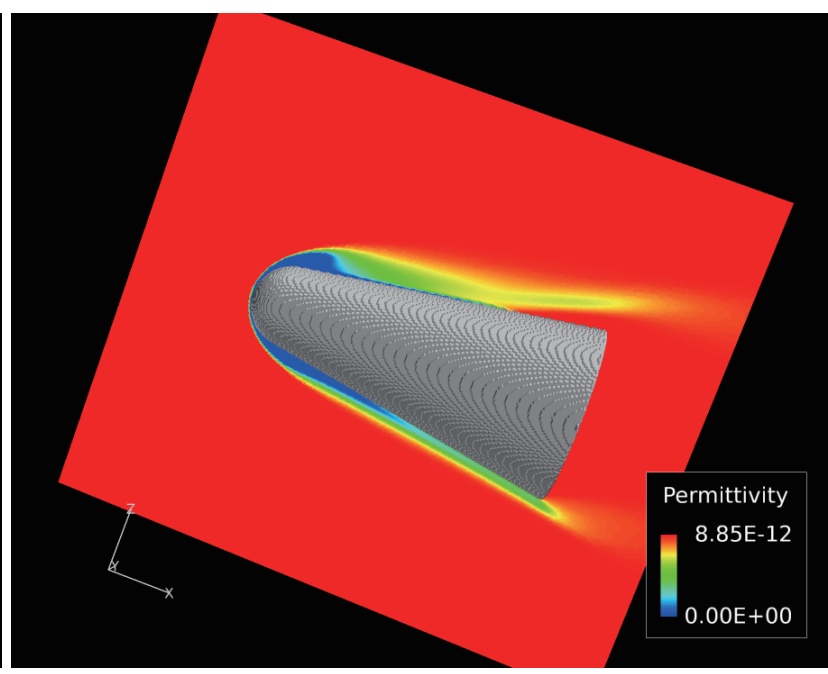

(b) altitude $70 \mathrm{~km}$.

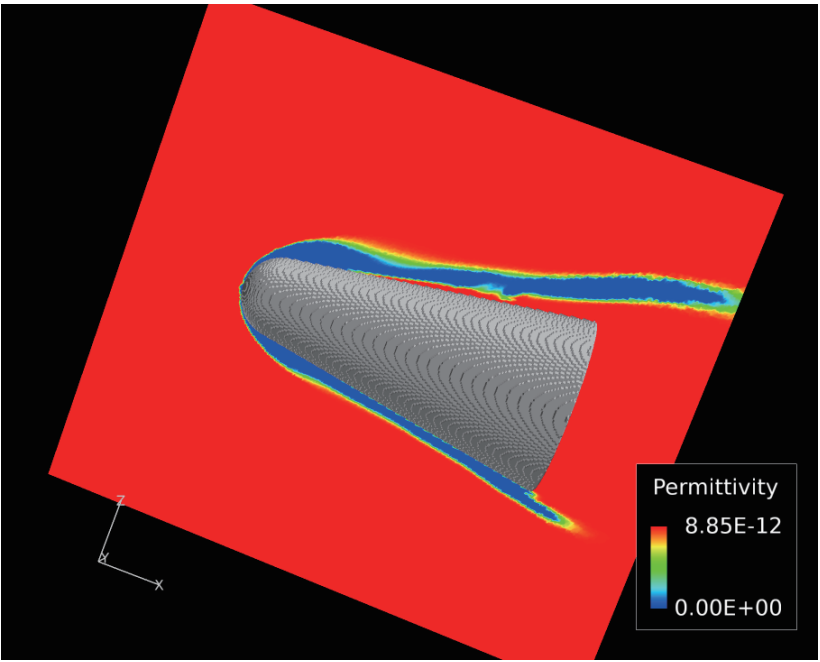

(d) altitude $50 \mathrm{~km}$.

Figure 8: Distributions of permittivity in $x-z$ plane $(y=0 \mathrm{~m})$ of FD2TD computational domain around RAM $\mathrm{C}$ vehicle at each altitude for C-band beacon horn $(5.80 \mathrm{GHz})$

Figures 8(a), 8(b), 8(c), and 8(d) show the distributions of the permittivity in the $x-z$ plane $(y=0 \mathrm{~m})$ of the FD2TD computational domain around the RAM C vehicle at the altitudes of $80,70,60$, and $50 \mathrm{~km}$, respectively. In these figures, the link angular frequency $(\omega)$ is set to that of the link frequency of the C-band: $f_{\text {cband }} / 2 \pi=9.23 \times 10^{7} \mathrm{rad} / \mathrm{s}$. A region where the permittivity is less than zero, caused by the strong aerodynamic heating, appears near the lower side surface of the vehicle at lower altitudes. On the other hand, in the upper region at the aft of the vehicle, highly dense plasma does not cover the surface because of flow separation. Although the plasma attenuation of the electromagnetic waves may be found in this region, the electromagnetic waves can propagate without reflection in this region.

Figures 9(a), 9(b), 9(c), and 9(d) show the electric field distributions of the electromagnetic waves in the $x$ - $z$ plane $(y=0 \mathrm{~m})$ of the FD2TD computational domain around the RAM C vehicle at the altitudes of $80,70,60$, and $50 \mathrm{~km}$, respectively, for the C-band beacon horn. The common logarithmic scale is used to illustrate the electric field distributions in these figures. The electromagnetic waves emitted from the antenna are observed to propagate in regions where the permittivity is high and the electron density is low, i.e., the rear of the 


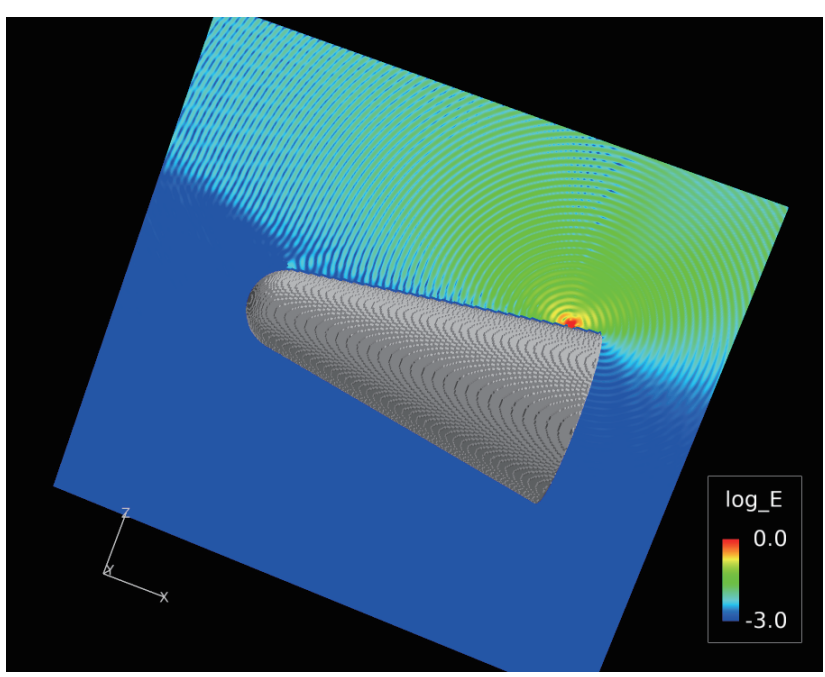

(a) altitude $80 \mathrm{~km}$.

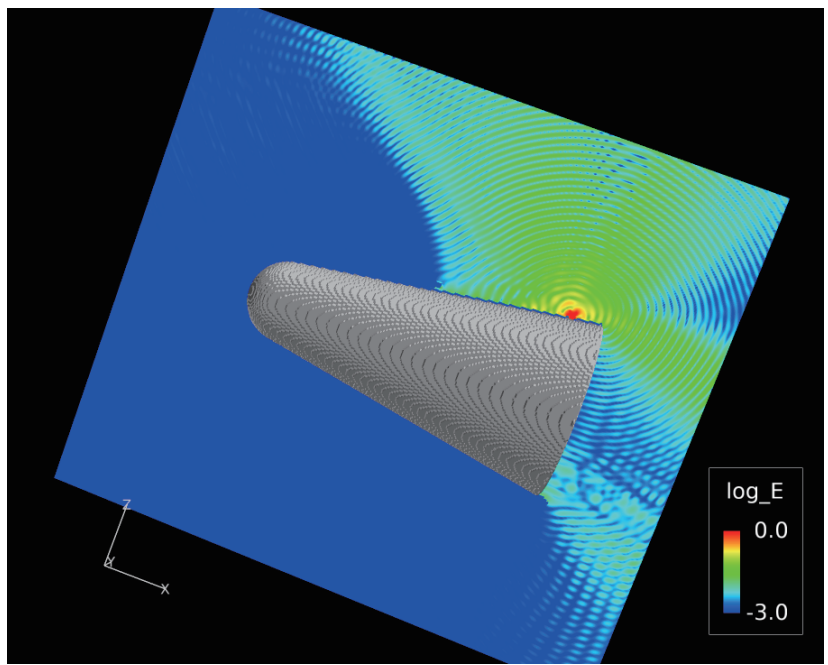

(c) altitude $60 \mathrm{~km}$.

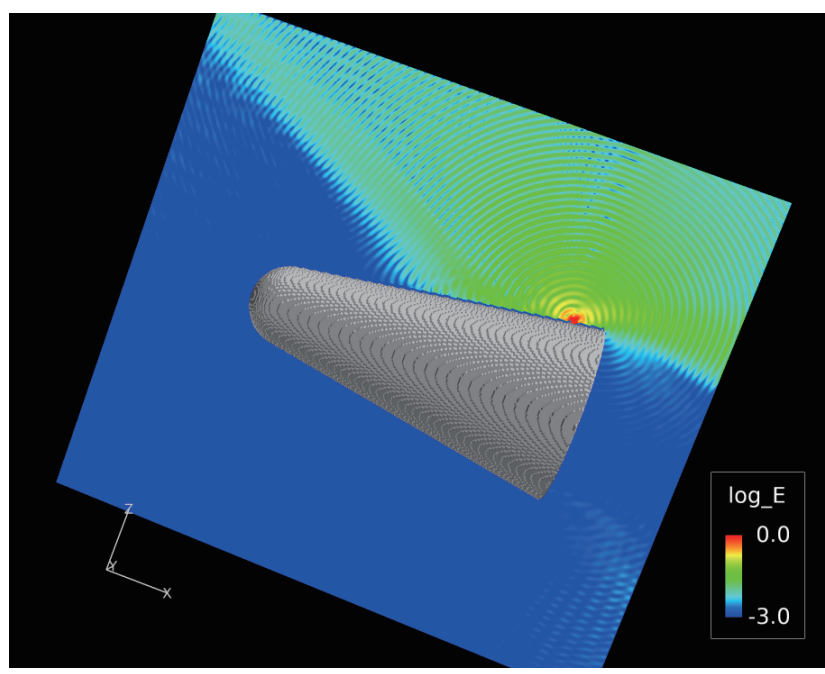

(b) altitude $70 \mathrm{~km}$.

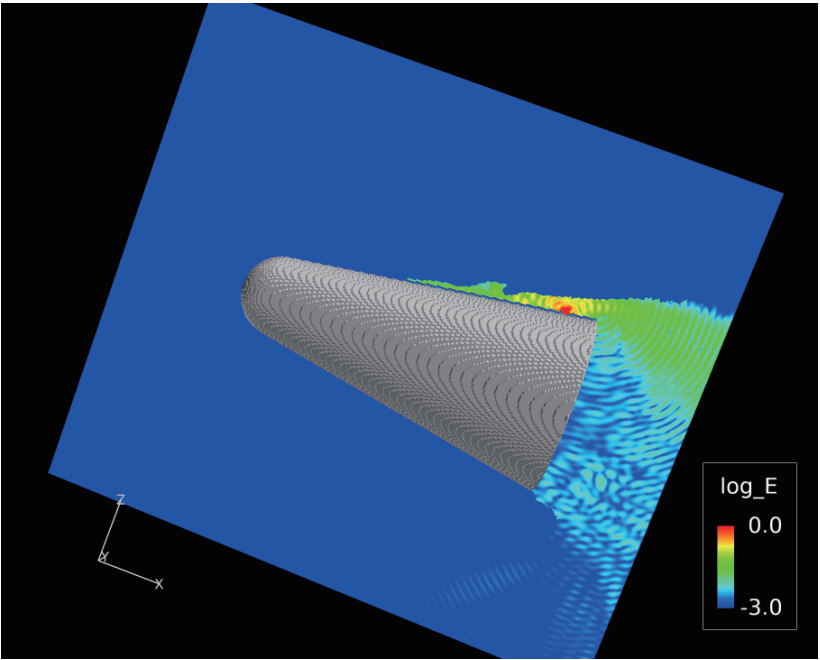

(d) altitude $50 \mathrm{~km}$.

Figure 9: Distributions of electric field in $x-z$ plane $(y=0 \mathrm{~m})$ of FD2TD computational domain around RAM $\mathrm{C}$ vehicle at each altitude for C-band beacon horn $(5.80 \mathrm{GHz})$

vehicle. On the other hand, the electromagnetic waves are reflected on the surface of the strong-shock layer. At the altitude of $80 \mathrm{~km}$, the critical number density of electrons distributes in a limited region in front of the vehicle, and the electromagnetic waves propagate without much reflection and attenuation. It is indicated that the reflection of the electromagnetic waves starts in the upper side region at the altitudes of 70 and $60 \mathrm{~km}$. At the altitude of $50 \mathrm{~km}$, highly dense plasma almost covers the surface, and the electromagnetic waves are intricately reflected with attenuation by the strongly conductive medium near the antenna. The electromagnetic waves cannot propagate to the front of the vehicle but only to the rear regions. If the RAM $\mathrm{C}$ vehicle is observed from the windward side, an $\mathrm{RF}$ blackout may be observed at this altitude. On the other hand, it is possible to detect an attenuated signal emitted from the vehicle at the leeward side of the vehicle, where a communication station is positioned.

In the RAM C flight experiments, plasma attenuation of the electromagnetic waves used for communication was measured with the C-band beacon and X-band telemetry horns. Because the RAM C-1 and RAM C-3 vehicles injected water into the plasma layer for reduction 


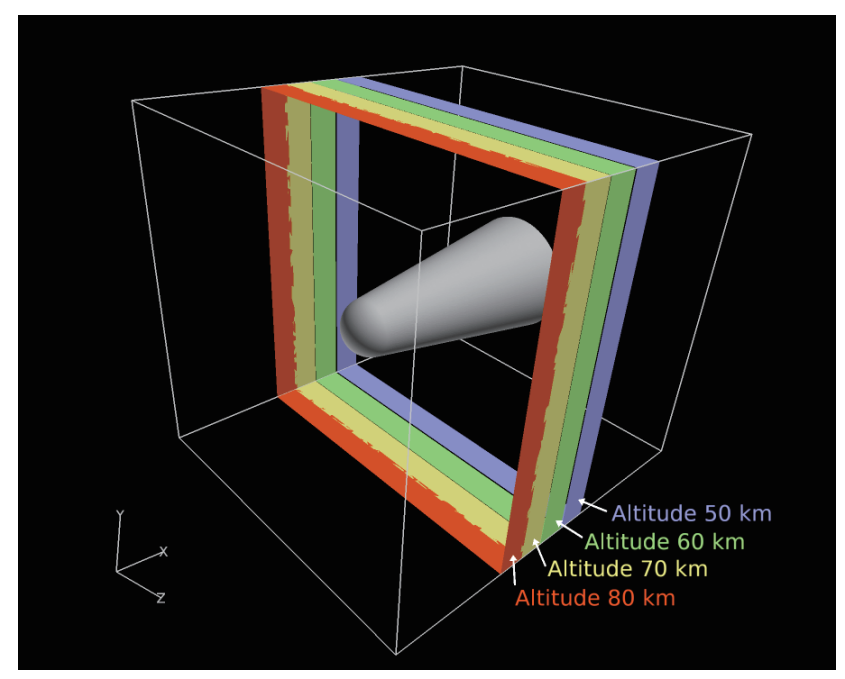

Figure 10: Windows in which the radar tracking the RAM $\mathrm{C}$ vehicle is visible at each altitude on the boundaries of the FD2TD domain

of the free electron level, it is supposed that the signal attenuations measured in these two flights were affected by the injection. In addition, the vehicles had a phenolic-graphite charring ablator on the nose caps. The ablator gas injected from the surface involving impurities can generally enhance the ionisation reaction of the plasma. On the other hand, the RAM C-2 injected no electrophile, such as water, during the reentry. To avoid contamination of the ablation gas, the RAM C-2 was also equipped with a beryllium heat sink on the nose cap until an altitude of $56 \mathrm{~km}$. Therefore, the signal loss history measured by the RAM C-2 vehicle is used in this study for validation of the analysis model of electromagnetic waves. In this simulation, the signal loss $G_{\mathrm{SL}}$ is calculated using the common logarithm, as follows:

$$
G_{\mathrm{SL}}=-10 \log \left(\frac{\left|\mathbf{E}_{b d}\right|^{2}}{\left|\mathbf{E}_{b d, \text { free }}\right|^{2}}\right) .
$$

where $\mathbf{E}_{b d}$ is the electric field vector of the electromagnetic waves passing the boundary face, and $\mathbf{E}_{b d, f r e e}$ is that of the electromagnetic waves though the boundary in a space where there is no plasma. The location of the Bermuda radar from the viewpoint of the RAM $\mathrm{C}$ vehicle during reentry can be obtained from Fig. 2. In addition, considering the RAM C flight with the angle of attack of $5^{\circ}$ and roll motion around the body axis, it is deduced that the windows in which the radar is visible at each altitude shown in Fig. 10 are on the boundaries of the FD2TD computational domain. Specifically, in the $x$-direction, the window ranges are set to $0.203-0.345 \mathrm{~m}$ at $80 \mathrm{~km}$ altitude, $0.348-0.518 \mathrm{~m}$ at $70 \mathrm{~km}$ altitude, $0.521-0.695 \mathrm{~m}$ at $60 \mathrm{~km}$ altitude, and $0.705-0.878 \mathrm{~m}$ at $50 \mathrm{~km}$ altitude. The electric fields used to evaluate the signal loss are calculated as the average values for these faces at each altitude.

Comparisons of the signal losses for the C-band and X-band between the experimental and computational results are shown in Fig. 11. Note that we refer to the studies by Schexnayder et al. and Huber et al. [14,39] for the measured signal losses. In the simulation, if attenuation of the electromagnetic waves occurs, the predicted signal loss becomes greater than zero. The measured signal losses for the C-band and X-band indicate that the attenuations by plasma started at altitudes of approximately $54 \mathrm{~km}$ and $51 \mathrm{~km}$, respectively. As the altitude decreased, steep increases in the signal loss appeared, and then the RF blackout phenomena 


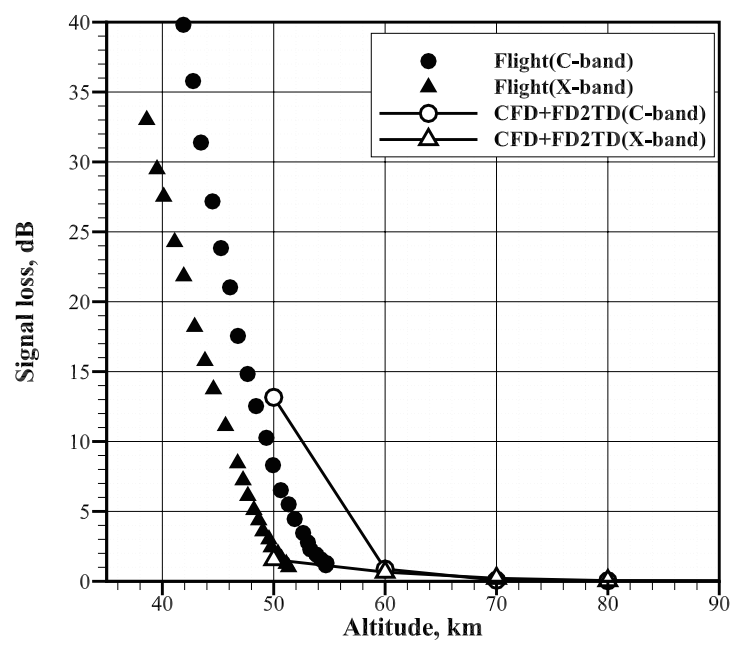

Figure 11: Comparisons of signal losses of electromagnetic waves for C-band and X-band antennas between flight data and computational results

were observed. Note that the RAM C vehicle recovered from the RF blackout at an altitude of approximately $25 \mathrm{~km}$ in the flight, although the recovery phase is not presented in the figure. The magnitude of the signal loss for the X-band is lower than that for the C-band, because of differences in the link frequency of the antennas. Generally, communication waves with higher frequencies propagate further in plasma than those with lower frequencies, according to the relation between critical electron density and frequency. This is the main reason why the X-band signal loss becomes lower, although the locations of both the antennas partly influence the tendency of the loss. The computed signal losses for the C-band and X-band show qualitatively good agreement with the trends of the measured data at all altitudes. This indicates that the starts of the RF blackout are reproduced well by the present model.

\section{Conclusions}

The plasma flow and the behaviour of electromagnetic waves around a reentry vehicle of the RAM project [14] during atmospheric reentry were numerically investigated for prediction of $\mathrm{RF}$ blackout and plasma attenuation. A formulation for the thermochemical non-equilibrium plasma flow during the aerodynamic heating was constructed, and its numerical implementation was achieved using the high-enthalpy flow simulation code RG-FaSTAR, which was developed according to the fast unstructured CFD solver "FaSTAR" [13] manufactured by JAXA. For an electromagnetic-wave simulation in plasma around reentry vehicle, the FD2TD method $[8,12]$ was used. The distributions of plasma properties obtained by the flow solver were transposed on the computational domain for the electromagnetic-wave simulation, and the behaviour of electromagnetic waves in plasma around the reentry vehicle was clarified. The computations were performed for four cases with altitudes between $80 \mathrm{~km}$ and $50 \mathrm{~km}$. In the flight experiments of the reentry vehicles, the profiles and peaks of the electron densities of the plasma layer were measured using the electrostatic probe and reflectometer techniques, in addition to the signal losses measured by the antennas mounted on the vehicle bodies. These data were used to validate the present combined simulation model. A comparison of 
the measured data and predicted results showed good agreement. It was suggested that the present combined method can be used to investigate the RF blackout for the effective design and development of reentry vehicles.

\section{Acknowledgments}

The author of the paper would like to thank Mr. Daniel Sors Raurell, The Technical University of Madrid, and Mr. Abhinav Kumar Jha, Indian Institute of Technology (currently, Bharat Petroleum Corporation Limited), for their valuable cooperation. This work was supported by JSPS KAKENHI Grant Number 26820366. The present computational results were obtained using the fast unstructured CFD code "FaSTAR", which was developed by the Japan Aerospace Exploration Agency (JAXA). The computations were mainly carried out using the computational facilities (HITACHI HA8000-tc/HT210 and FUJITSU PRIMERGY CX400) at the Research Institute for Information Technology, Kyushu University, and the supercomputer system (HITACHI SR16000 model M1) at the Information Initiative Center, Hokkaido University.

\section{References}

[1] J.P. Rybak and R.J. Churchill. "Progress in Reentry Communications". IEEE Transctions on Aerospace and Electorinic System, 7(5):879-894, September 1971.

[2] L.C. Schroeder and F.P. Russo. "Flight Investigation and Analysis of Alleviation of Communications Blackout by Water Injection during Gemini 3 Reentry". NASA TM $X$-1521, pages 1-56, 1968.

[3] M. Kim, M. Keidar, and I.D. Boyd. "Analysis of an Electromagnetic Mitigation Scheme for Reetnry Telemetry Through Plasma". Journal of Spacecraft and Rockets, 45(6):12231229, November-December 2008.

[4] M. Kundrapu, J. Loverich, K. Beckwith, and P. Stoltz. "Modeling Radio Communication Blackout and Blackout Mitigation in Hypersonic Vehicles". Journal of Spacecraft and Rockets, 52(3):853-862, May 2015.

[5] H. Usui, H. Matsumoto, F. Yamashita, M. Yamane, and S. Takenaka. "Computer Experiments on Radio Blackout of a Reentry Vehicle". In D.L. Cooke, editor, Proceedings of 6th Spacecraft Charging Technology Conference, AFRL-VS-TR-20001578, pages 107110. Eurpean Space Agency, the NASA Astrophysics Data System, September 2000.

[6] I.F. Belov, V.Y. Borovoy, V.A. Gorelov, A.Y. Kireev, A.S. Korolev, and E.A. Stepanov. "Investigation of remote antenna assembly for radio communication with reentry vehicle". Journal of Spacecraft and Rockets, 38(2):249-256, 2001.

[7] Y. Takahashi, K. Yamada, and T. Abe. "Radio Frequency Blackout Possibility for an Inflatable Reentry Vehicle". AIAA Paper 2012-3110, 2012.

[8] Y. Takahashi, K. Yamada, and T. Abe. "Examination of Radio Frequency Blackout for an Inflatable Vehicle during Atmospheric Reentry". Journal of Spacecraft and Rockets, 51(2):430-441, March 2014. 
[9] K.S. Kunz and R.J. Luebbers. The Finite Difference Time Domain Method for Electromagnetics. CRC Press, Boca Raton, 1993.

[10] K. Kinefuchi, I. Funaki, and T. Abe. "Frequency-Dependent FDTD Simulation of the Interaction of Microwaves With Rocket-Plume". IEEE Transactions on Antennas and Propagation, 58(10):3282-3288, October 2010.

[11] S.A. Cummer. "An Analysis of New and Existing FDTD Methods for Isotropic Cold Plasma and a Method for Improving Their Accuracy". IEEE Transactions on Antennas and Propagation, 45(3):392-400, March 1997.

[12] Y. Takahashi, K. Yamada, and T. Abe. "Prediction Performance of Blackout and Plasma Attenuation in Atmospheric Reentry Demonstrator Mission". Journal of Spacecraft and Rockets, 51(6):1954-1964, Novermber 2014.

[13] A. Hashimoto, K. Murakami, T. Aoyama, K. Ishiko, M. Hishida, M. Sakashita, and P.R. Lahur. "Toward the Fastest Unstructured CFD". AIAA Paper 2012-1075, 2012.

[14] P.E. Huber, J.S. Evans, and C.J. Schexnayder Jr. "Comparison of Theoretical and Flight-Measured Ionization in a Blunt Body Re-Entry Flowfield". AIAA Journal, 9(6):1154-1162, 1971.

[15] W.L. Weaver and J.T. Bowen. "Entry Trajectory, Entry Environment, and Analysis of Spacecraft Motion for the RAM C-III Flight Experiment". NASA TM X-2562, pages $1-95,1972$.

[16] J.M. Yos. "Transport Properties of Nitrogen, Hydrogen Oxygen and Air to 30,000 K". TRAD-TM-63-7, Research and Advanced Development Division, AVCO Corp., 1963.

[17] C.F. Curtiss and J.O. Hirschfelder. "Transport Properties of Multicomponent Gas Mixture". Journal of Chemical Physics, 17(6):550-555, June 1949.

[18] R.N. Gupta, J.M. Yos, R.A. Thompson, and K.P. Lee. "A Review of Reaction Rates and Thermodynamic and Transport Properties for an 11-Species Air Model for Chemical and Thermal Nonequilibrium Calculations to 30000 K". NASA RP-1232, Aug. 1990.

[19] M. Fertig, A. Dohr, and H.H. Frühaufu. "Transport Coefficients for High-Temperature Nonequilibrium Air Flows". AIAA Paper 98-2937, 1998.

[20] M. Fertig, A. Dohr, and H.H. Frühauf. "Transport Coefficients for High-Temperature Nonequilibrium Air Flows". Journal of Thermophysics and Heat Transfer, 15(2):148156, April-June 2001.

[21] C. Park. "Assessment of a Two-Temperature Kinetic Model for Dissociating and Weakly Ionizing Nitrogen". Journal of Thermophysics and Heat Transfer, 2(1):8-16, Jan.-March 1988.

[22] C. Park. Nonequilibrium Hypersonic Aerothermodynamics. Wiley, New York, 1990.

[23] J.G. Parker. "Rotational and Vibrational Relaxation in Diatomic Gases". The Physics of Fluids, 2(4):449-462, July-Aug. 1959. 
[24] C. Park. "Rotational Relaxation of $\mathrm{N}_{2}$ Behind a Strong Shock Wave". Journal of Thermophysics and Heat Transfer, 18(4):527-533, Oct.-Dec. 2004.

[25] R.C. Millikan and D.R. White. "Systematics of Vibrational Relaxation". The Journal of Chemical Physics, 39(12):3209-3213, Dec. 1963.

[26] C. Park. "Problems of Rate Chemistry in the Flight Regimes of Aeroassisted Orbital Transfer Vehicles". AIAA Paper 84-1730, 1984.

[27] J.P. Appleton and K.N.C. Bray. "The Conservation Equations for a Nonequilibrium Plasma". Journal of Fluid Mechanics, 20(4):659-672, June 1964.

[28] M. Mitchner and C.H. Kruger Jr. Partially Ionized Gases. Wiley, New York, 1973.

[29] P.A. Gnoffo, R.N. Gupta, and J.L. Shinn. "Conservation Equations and Physical Models for Hypersonic Air Flows in Thermal and Chemical Nonequilibrium". NASA TP-2867, Feb. 1989.

[30] M. Nishida and M. Matsumoto. "Thermochemical Nonequilibrium in Rapidly Expanding Flows of High-Temperature Air". Zeitschrift für Naturforcuhung, Teil A: Physik, Physikalische Chemie, Kosmophysik, 52(4):358-368, 1997.

[31] S.S. Lazdinis and S.L. Petrie. "Free Electron and Vibrational Temperature Nonequilibrium in High Temperature Nitrogen". Physics of Fluids, 17(8):1539-1546, Aug. 1974.

[32] J.H. Lee. "Electron-Impact Vibrational Relaxation in High-Temperature Nitrogen". Journal of Thermophysics and Heat Transfer, 7(3):399-405, July-Sept. 1993.

[33] C. Park and S.H. Lee. "Validation of Multitemperature Nozzle Flow Code". Journal of Thermophysics and Heat Transfer, 9(1):9-16, Jan.-Mar. 1995.

[34] E. Shima and K. Kitamura. "Parameter-Free Simple Low-Dissipation AUSM-Family Scheme for All Speeds". AIAA Journal, 49(8):1693-1709, August 2011.

[35] A. Jameson and S. Yoon. "Lower-Upper Implicit Schemes with Multiple Grids for the Euler Equations". AIAA Journal, 25(7):929-935, July 1987.

[36] T.R.A. Bussing and E.M. Murman. "Finite-Volume Method for the Calculation of Compressible Chemically Reacting Flows". AIAA Journal, 26(9):1070-1078, 1988.

[37] W.L. Jones Jr. and A.E. Cross. "Electrostatic Probe Measurement of Plasma Surrounding Three 25000 Foot per Second Reentry Flight Experiments". The Entry Plasma Sheath and its Effect on Space Vehicle Electromagnetic Systems, NASA SP-252, pages 109-136, 1971.

[38] G. Mur. "Absorbing Boundary Conditions for the Finite-Difference Approximation of the Time-Domain Electromagnetic-Field Equations". IEEE Transactions on Electromagnetic Compatibility, EMC-23(4):377-382, 1981.

[39] C.J. Schexnayder Jr., J.S. Evans, and P.E. Huber. "Comparison of Theoretical and Experimental Electron Density for RAM C Flights". The Entry Plasma Sheath and its Effect on Space Vehicle Electromagnetic Systems, NASA SP-252, pages 277-303, 1971. 